\title{
The DNA damage checkpoint precedes activation of ARF in response to escalating oncogenic stress during tumorigenesis
}

\author{
K Evangelou ${ }^{1,12}$, J Bartkova ${ }^{2,12}$, A Kotsinas ${ }^{1,12}$, IS Pateras ${ }^{1,12}$, M Liontos ${ }^{1}$, G Velimezi ${ }^{1}$, M Kosar ${ }^{2,3}$, T Liloglou ${ }^{4}$, IP Trougakos ${ }^{5}$,

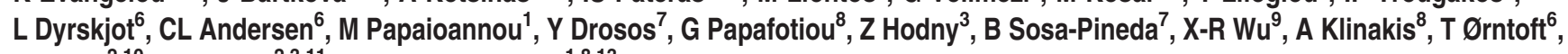 \\ $\mathrm{J}_{\text {Lukas }}^{2,10}$, J Bartek ${ }^{\star, 2,3,11}$ and VG Gorgoulis ${ }^{\star, 1,8,13}$
}

Oncogenic stimuli trigger the DNA damage response (DDR) and induction of the alternative reading frame (ARF) tumor suppressor, both of which can activate the p53 pathway and provide intrinsic barriers to tumor progression. However, the respective timeframes and signal thresholds for ARF induction and DDR activation during tumorigenesis remain elusive. Here, these issues were addressed by analyses of mouse models of urinary bladder, colon, pancreatic and skin premalignant and malignant lesions. Consistently, ARF expression occurred at a later stage of tumor progression than activation of the DDR or p16 ${ }^{I N K 4 A}$, a tumor-suppressor gene overlapping with ARF. Analogous results were obtained in several human clinical settings, including early and progressive lesions of the urinary bladder, head and neck, skin and pancreas. Mechanistic analyses of epithelial and fibroblast cell models exposed to various oncogenes showed that the delayed upregulation of ARF reflected a requirement for a higher, transcriptionally based threshold of oncogenic stress, elicited by at least two oncogenic 'hits', compared with lower activation threshold for DDR. We propose that relative to DDR activation, ARF provides a complementary and delayed barrier to tumor development, responding to more robust stimuli of escalating oncogenic overload.

Cell Death and Differentiation (2013) 20, 1485-1497; doi:10.1038/cdd.2013.76; published online 12 July 2013

\section{Introduction}

Oncogenic stimuli elicit cellular responses that commonly lead to premature senescence or cell death, depending on the cell type, nature and intensity of deleterious signals. ${ }^{1-4}$ Suppression of cell death and senescence is imperative for cancer progression. ${ }^{1,4}$ The p53 protein has an established role in imposing the apoptotic and senescence antitumor barriers. ${ }^{1,2,4-6}$ Nevertheless, the relative contribution of two major routes known to trigger p53 during oncogenic challenge, the DNA damage response (DDR) network ${ }^{7-11}$ and the alternative reading frame (ARF) tumor suppressor, $2,4,5,12,13$ remains unexplored.

DDR detects various DNA lesions, signals their presence and promotes appropriate cellular response. ${ }^{14-16}$ DDR signalling is initiated upon binding of sensor complexes at sites of DNA damage, followed by recruitment of ATM and ATR kinases that phosphorylate various cellular proteins including the Chk2- and Chk1-kinases, respectively. The ATM/Chk2 and ATR/Chk1 modules regulate downstream effectors, including p53 that is pivotal for the cellular response to genotoxic insults and other stress types. ${ }^{15}$

The second pathway involves ARF expression, ${ }^{13}$ whose gene resides in CDKN2 locus, overlapping with the $\mathrm{p} 16^{\mathrm{INK} 4 \mathrm{~A}}$ tumor-supressor and cell-cycle inhibitor gene. ${ }^{13,17}$ ARF antagonizes MDM2, a p53 specific E3-ubiquitin ligase, stabilizing p53 and enhancing its activity.

Although there is substantial evidence to support that oncogenic stress can activate both DDR and ARF, ${ }^{1,2,12}$

\footnotetext{
${ }^{1}$ Molecular Carcinogenesis Group, Department of Histology and Embryology, School of Medicine, University of Athens, Athens, Greece; ${ }^{2}$ Danish Cancer Society, Copenhagen, Denmark; ${ }^{3}$ Laboratory of Genome Integrity, Institute of Molecular Genetics, Academy of Science of the Czech Republic, Prague, Czech Republic; ${ }^{4}$ Molecular Oncology Unit, Roy Castle International Centre for Lung Cancer Research, Liverpool, UK; ${ }^{5}$ Department of Cell Biology and Biophysics, Faculty of Biology, University of Athens, Athens, Greece; ${ }^{6}$ Department of Molecular Medicine, University Hospital, Aarhus, Denmark; ${ }^{7}$ Department of Genetics, St. Jude Children's Research Hospital, Memphis, TN, USA; ${ }^{8}$ Basic Research Center, Biomedical Research Foundation, Academy of Athens, Athens, Greece; ${ }^{9}$ Department of Urology and Pathology, New York University School of Medicine and Veterans Affairs Medical Center in Manhattan, New York, USA; ${ }^{10}$ Novo Nordisk Foundation Center for Protein Research, Disease Biology, University of Copenhagen, Copenhagen, Denmark and ${ }^{11}$ Institute of Molecular and Translational Medicine, Palacky University, Olomouc, Czech Republic

${ }^{*}$ Corresponding author: J Bartek, Danish Cancer Society Research Center, Strandboulevarden 49, DK-2100 Copenhagen, Denmark. Tel: + 4535257357 ; Fax: + 453527 1811; E-mail: jb@ cancer.dk

or VG Gorgoulis, Molecular Carcinogenesis Group, Department of Histology and Embryology, University of Athens, School of Medicine, Medical School, NKUA, 75 Mikras Asias Street, Goudi, Athens GR-11146, Greece. Tel: +30 210746 2352; Fax: + 30210746 2340; E-mail: vgorg @ med.uoa.gr

${ }^{12}$ These authors contributed equally to this work.

${ }^{13}$ Dedicated to the memory of George V Gorgoulis.

Keywords: ARF; carcinogenesis; DDR; E2F1; p16 $6^{\text {INK4A }}$

Abbreviations: AOM, azoxymethane; ARF, alternative reading frame; ATM-p, phosphorylated at Ser1981 ATM; ChIP, chromatin immunoprecipitation; DABG, detection above background; DDR, DNA damage response; DMEM, Dulbecco's modified Eagle's medium; DSS, dextran-sodium-sulfate; Ha-Ras Q61L, amino-acid substitution at position 61 in Ha-RAS from glutamine $(\mathrm{Q})$ to leucine (L); HBEC, human bronchial epithelial cell; hTERT, human telomerase reverse transcriptase; Ncstn ${ }^{-1-}$, homozygous conditional nicastrin deletion; PanIN, pancreatic intraepithelial neoplasia; pT68-Chk2, phosphorylated at Thr68 Chk2; Sa- $\beta$-gal, senescence associated $\beta$-galactosidase; siRNA, small interfering RNA; $\gamma \mathrm{H} 2 \mathrm{AX}$, phosphorylated at Ser139 Histone H2AX

Received 18.5.13; revised 28.5.13; accepted 29.5.13; Edited by G Melino; published online 12.7.13
} 
the key issue of the relationship between these anticancer barriers and other questions in this field remain unresolved. For example, are DDR and ARF triggered concurrently or at different cancer developmental stages? Is the activation of DDR versus ARF dependent on cell/tissue type, species or a specific oncogene and/or the 'strength' of an oncogenicinsult? Another issue is that of a potential 'hierarchy' of response within the CDKN2 locus that is frequently targeted during cancer, that is, the question of which of the two tumor suppressors, ARF or $\mathrm{p} 16^{\mathrm{INK} 4 \mathrm{~A}}$, is activated preferentially, given that cancer-associated deletions of this locus often affect both genes. ${ }^{2,13,17}$

Considering these questions, we investigated the above pathways in clinical settings and in vivo mouse tumor models covering the entire histopathological spectrum of cancer development, complemented by functional analyses of human cellular models exploring various oncogenic stimuli.

\section{Results}

The DDR pathway is activated earlier than ARF during progression of murine premalignant lesions to malignancy. The relationship between DDR activation and ARF induction was examined in various experimental mouse models. In most cases, the entire pathological sequence of cancer progression was available.

The first model involved a Ha-Ras transgenic mouse that expressed, under the control of mouse uroplakin-II promoter, a mutant Ha-Ras (codon Q61L) in the urothelium. ${ }^{18}$ Heterozygous (Ha-Ras ${ }^{L 61+/-}$ ) mice developed urothelial hyperproliferation and simple hyperplasias. In contrast, homozygous (Ha-Ras ${ }^{L 61+/+}$ ) mice developed nodular hyperplasias, which later progressed to low-grade, noninvasive superficial papillary tumors. Immunohistochemical examination of DDR status with various validated antibodies revealed activation from simple urothelial hyperplasia, as demonstrated by phosphorylation of histone-H2AX $(\gamma \mathrm{H} 2 \mathrm{AX})$ and Chk2 (phosphorylated at Thr68 Chk2). Conversely, ARF expression studied on parallel sections appeared later, at tumor stage that required two mutant Ha-Ras alleles (Figure 1a). The percentage of ARF-positive cells, even at the tumor stage, was also overall lower than that of DDR-positive cells (Figure 1a).

Next model comprised the azoxymethane (AOM) and dextran sodium sulfate (DSS)-induced colitis, which progressed to cancer. ${ }^{19}$ Within this context $K$-Ras and Wnt signalling pathways become activated ${ }^{20}$ and $\mathrm{p} 16^{\text {INK4A }}$ expression is increased from preneoplastic stages. ${ }^{21}$ This model resembles the human idiopathic inflammatory bowel disease, a condition with increased risk for developing cancer. ${ }^{22}$ Preneoplastic and neoplastic lesions obtained from these mice covered the whole spectrum of carcinogenesis, ranging from atypical regenerative hyperplasias to adenomas, and adenocarcinomas. In situ analysis revealed activated DDR, depicted by nuclear $\gamma \mathrm{H} 2 \mathrm{AX}$ and phosphorylated ATM kinase, in all stages of cancer development. In contrast to early DDR activation, ARF expression was observed in later stages (Figure $1 \mathrm{~b}$ ). Relevant to this, the $p 16^{I N K 4 A}$ gene can be methylated early, whereas $A R F$ is not affected. ${ }^{23}$
In the third model, precancerous lesions were induced by conditional expression of K-Ras ${ }^{D 12}$ in the pancreas, recapitulating the human preneoplastic (pancreatic intraepithelial neoplasia) PanIN stages 1-3. No adenocarcinomas were developed in this setting. DDR markers were robustly expressed at all PanINs 1-3, whereas ARF was detected only in PanINs 2-3 (Supplementary Figure 1a). Notably, p16 ${ }^{\text {INK4A }}$ expression was decreased in PanINs 2-3, in accordance with data from human PanINs showing specific $p 16^{\text {INK4A }}$ methylation from PanIN-1 to ductal adenocarcinoma, in contrast to $p 14^{A R F}$ gene that remained unmethylated in this setting, thereby supporting specific selection toward p16 $6^{I N K 4 A}$ inactivation early in pancreatic tumorigenesis. ${ }^{24}$

The fourth model carried a homozygous conditional nicastrin $\left(\mathrm{Ncstn}^{-/-}\right)$deletion in the mouse skin. Nicastrin is a subunit of the $\gamma$-secretase complex, responsible for Notch1receptor maturation. ${ }^{25}$ Hence, the model resembles a conditional Notch1 deletion. ${ }^{26}$ Low-grade and high-grade skin dysplasias were produced. Consistent with the other mouse models (Figure 1; Supplementary Figure 1) the DDR markers were expressed intensely in all skin lesions, whereas increased ARF was detected only in high-grade dysplasias (Supplementary Figure 1b).

DDR is activated earlier than ARF during human epithelial tumorigenesis. Next, we examined the relationship between DDR and ARF expression during cancer progression in human clinical samples. We employed panels with major stages of head and neck, urinary bladder and skin cancer development. A limited number of human pancreatic cancers encompassing all pathological stages of development was also examined.

In human head and neck and urinary bladder lesions, DDR signalling appeared massively activated from the early stages (hyperplasias and superficial Ta-lesions, respectively), as demonstrated by $\mathrm{H} 2 \mathrm{AX}$ and ATM, or Chk2, phosphorylation (Figures 2a, bi and bii). Heterogeneous expression of p53 along with its $\mathrm{p} 21^{\mathrm{WAF} 1}$ target was evident in early stages, indicating that wild-type p53 is activated in these lesions. However, as lesions progressed p21 WAF1 expression decreased, whereas the frequency and abundance of p53 expression was often enhanced compared with earlier lesions (Figure 2b). This 'discrepancy' between low/absent p21 WAF1 and high p53 levels at later stages of tumor progression is consistent with frequent p53 mutations in most tumors. ${ }^{27,28}$ Contrary to the observed DDR activation, ARF levels were virtually absent in early stages and increased gradually in advanced tumors, as confirmed immunohistochemically with three antibodies (Figures 2a and b, Supplementary Figure 2, and data not shown). Importantly, the observed trend from absent/low toward higher ARF expression with advancing tumor progression in head and neck and urinary bladder lesions was also detected at mRNA level (Figures 2aii and 2bii; Supplementary Tables 1 and 2). Overall, the DDR activation preceded both the induction of ARF and overabundance/mutation of $\mathrm{p53}$, the latter consistent with selection for p53 mutations after it had been activated by upstream DDR signalling. ${ }^{7,8}$

Contrary to ARF, p16 ${ }^{\text {INK4A }}$ was expressed in the precancerous and cancerous lesions, being occasionally detectable 


\section{a}

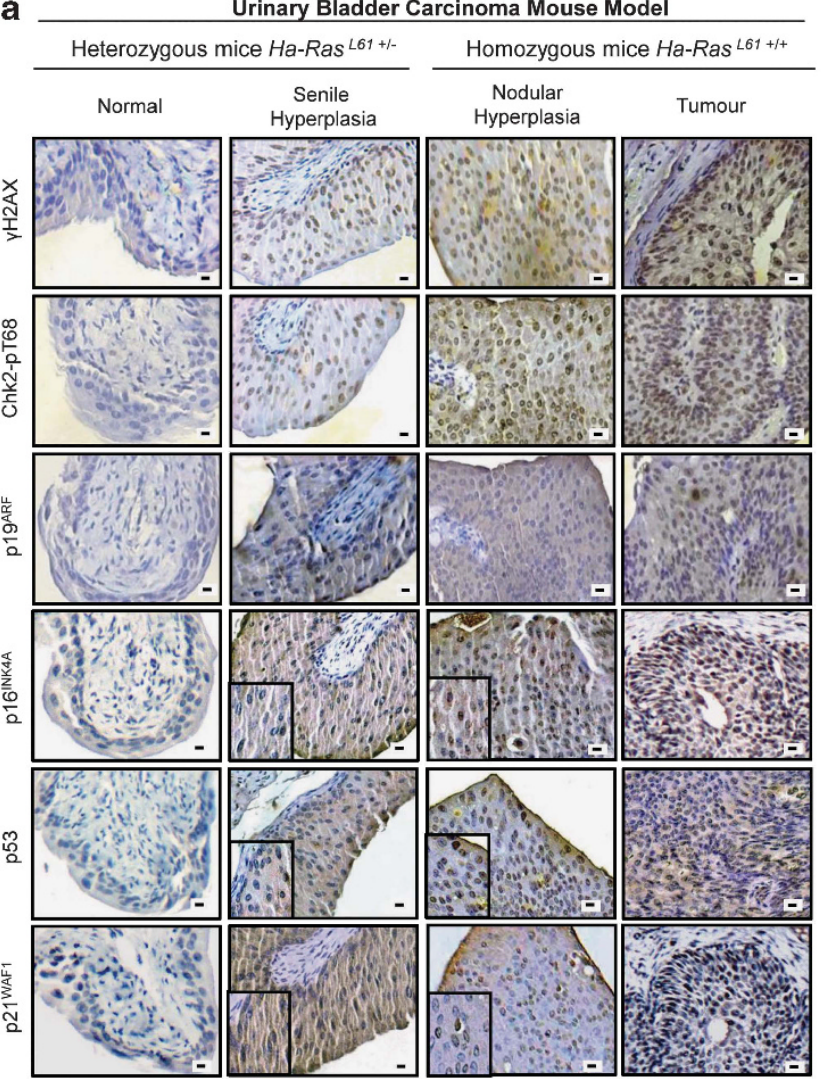

b

AOM/DSS Colitis-Associated Carcinoma Mouse Model
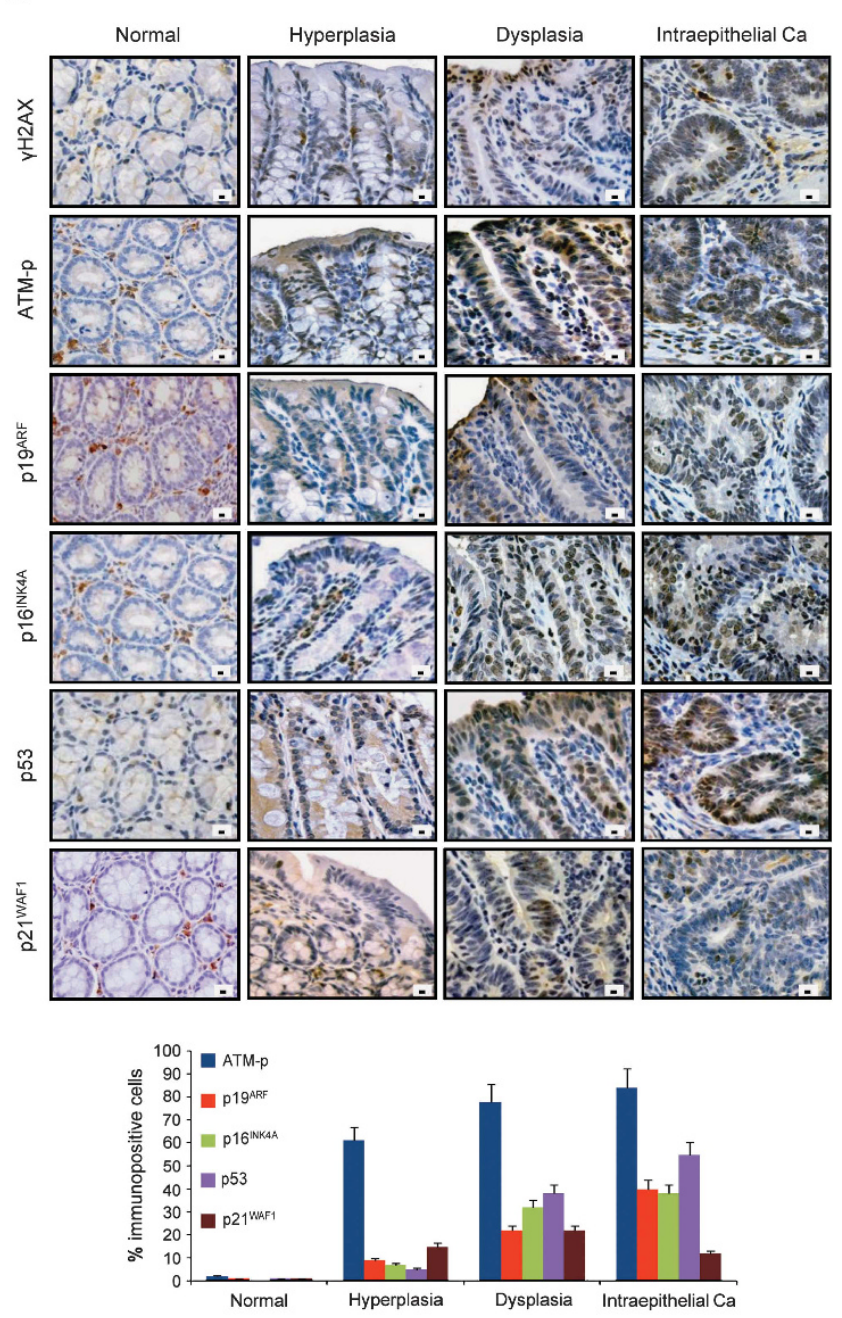

Figure 1 Activation of the DDR pathway precedes ARF upregulation during murine carcinogenesis. Immunohistochemical analysis of activated DDR markers $(\gamma \mathrm{H} 2 \mathrm{AX}$, Chk2-pT68, ATM-p), p19 ARF, p16 $6^{\text {INK4A }}$, p53 and p21 ${ }^{\text {WAF1 }}$ in bladder tumors and precancerous urothelial lesions from UPII/Ha-Ras ${ }^{\text {G12V }}$ transgenic mice (a) and precancerous lesions and colon adenocarcinomas from the colonic epithelium of AOM/DSS-treated mice (b). Corresponding histograms denote the average percentage expression of each molecule analyzed in normal tissues, precancerous lesions and carcinomas, respectively. Scale $=50 \mu \mathrm{m}$

in morphologically normal epithelium of head and neck samples, and more apparent in early lesions of head and neck and bladder origin (Figure 2). The CDKN2 locus is known to be targeted early in both bladder and head and neck cancer. ${ }^{29,30}$ Indeed, we found CDKN2A inactivation in a subset of our cases, as shown by DNA copy number and lossof-heterozygosity analysis at various progression stages of human urinary bladder and head and neck tumors (Figures 2biii and biv; Supplementary Figures 3 and 4; Supplementary Tables 1 and 2). Given that our genetic, protein and mRNA analyses showed that in the large proportion of tumors with preserved CDKN2 locus ARF becomes upregulated only later during progression of bladder tumors, our data indicate that the observed CDKN2A loss in earlier stages of carcinogenesis reflects selection against $\mathrm{p} 16^{\mathrm{INK} 4 \mathrm{~A}}$, rather than ARF.

Analogous results were obtained in human pancreatic lesions (Supplementary Figure 5). Characteristically, all cases harbored K-Ras mutations from PanIN-1, resembling the pancreatic murine model described above (Supplementary Figure 1a).

Finally, a panel of squamous cell skin carcinomas including adjacent in situ carcinomas (Bowen disease) and actinic keratosis lesions was investigated. Actinic keratosis and Bowen disease are sunlight-related disorders, considered as precursor lesions of squamous cell skin carcinomas. ${ }^{31}$ In these settings, DDR and ARF immunohistochemical analyses revealed activation of both from the earliest lesions (actinic keratoses), although DDR activation was more prominent (Supplementary Figure 6a). The early ARF induction in this context may be related to the fact that squamous cell skin carcinoma is sunlight related and the INK4A/ARF locus appears to have a role in UV-induced stress. ${ }^{32}$

Consistent with the latter, in a human skin xenograft model, in which hyperplasias were induced by adenoviral-mediated expression of growth factors (basic Fibroblast Growth Factor, Stem Cell Factor and Endothelin) and were UV unrelated, 
a i
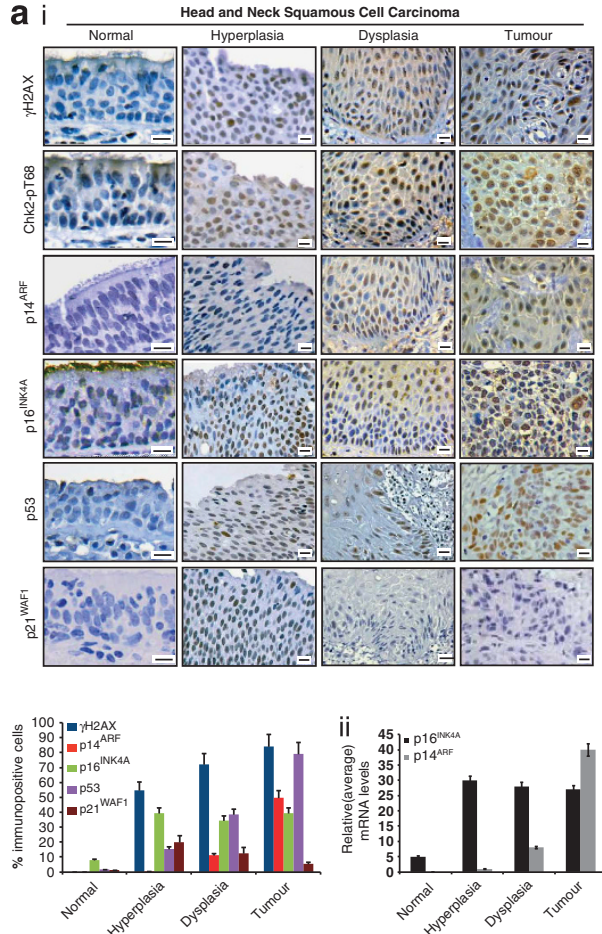

b i
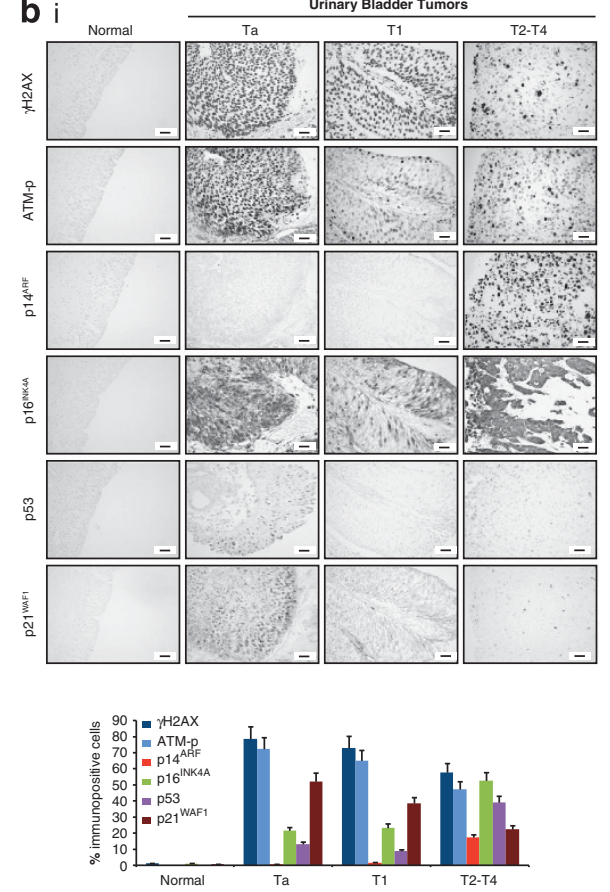

ii
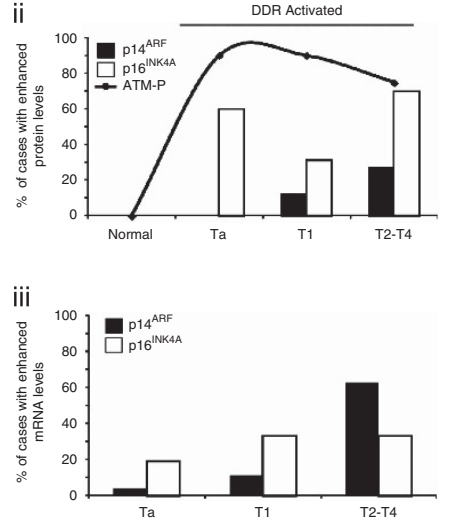

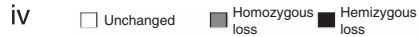

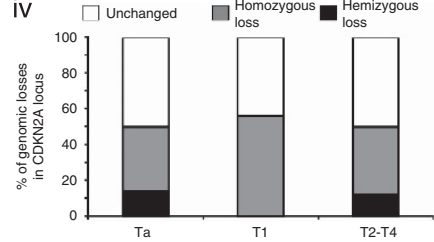

Figure 2 Activation of the DDR pathway precedes ARF upregulation during human head and neck (a) and human urinary bladder carcinogenesis (b). Immunohistochemical analysis for DDR markers ( $\gamma \mathrm{H} 2 \mathrm{AX}$, Chk2-pT68), p53, p2 $1^{\mathrm{WAF} 1}, \mathrm{p} 14^{\mathrm{ARF}}$ and p16 ${ }^{\mathrm{INK} 4 \mathrm{~A}}$ in normal laryngeal epithelium, corresponding hyperplastic and dysplastic lesions as well as head and neck carcinomas from the same patient (depicted case does not exhibit D9S171 LOH) (ai). Bars represent the average percentage expression for each protein in normal tissue, hyperplastic, dysplastic and tumor areas, respectively (ai). mRNA analysis in the same head and neck cases. Histogram shows average $\mathrm{p} 14^{\mathrm{ARF}}$ (gray bars) and $\mathrm{p} 16^{\mathrm{INK} 4 \mathrm{~A}}$ (black bars) mRNA levels in normal, hyperplastic and dysplastic epithelium and in tumor regions, respectively (aii). Representative images and bar graphs depicting the percentage of human urinary bladder cancer cases with enhanced expression (bi, bii) based on the immunohistochemical analysis for DDR markers $(\gamma \mathrm{H} 2 \mathrm{AX}, \mathrm{ATM}-\mathrm{p}), \mathrm{p} 53, \mathrm{p} 21^{\mathrm{WAF} 1}, \mathrm{p} 14^{\mathrm{ARF}}$ and $\mathrm{p} 16^{\mathrm{INK} 4 \mathrm{~A}}$ in normal bladder epithelium, preneoplastic lesions (Ta), non-invasive (T1) and invasive bladder carcinomas (T2-T4). (biii) Bar graphs showing the percentage of cases with increased p14 ${ }^{\text {ARF }}$ and p16 ${ }^{\text {INK4A }}$ mRNA levels in precancerous lesions (Ta) and bladder carcinomas either non-invasive (T1) or invasive (T2-T4). (biv) Cumulative bars showing the percentage of cases analyzed with losses at the CDKN2A locus at the various stages of urinary bladder carcinogenesis. Scale: $a=50 \mu \mathrm{m}, \mathrm{b}=100 \mu \mathrm{m}$

pronounced DDR activation was observed, contrary to undetectable ARF levels (Supplementary Figure 6b).

Higher threshold is necessary for oncogene-induced ARF expression than that required for DDR activation. Data so far showed almost exclusive activation of DDR in premalignant stages of epithelial carcinogenesis. As many of these early lesions eventually progress, expression of ARF, predominantly, at later stages of carcinogenesis (Figures 1 and 2, Supplementary Figures 1-6) implies that ARF may probably represent a delayed or 'secondary' anti-oncogenic response, complementary to DDR. An emerging question is why ARF 'does not sense and/or respond to' oncogenic signals that occur during initial steps of cancer development. A plausible scenario is that ARF activation requires a higher oncogenic threshold than DDR.

To explore this, we studied human bronchial epithelial cells (HBECs), into which various oncogenes were sequentially introduced. Transient Ha-Ras ${ }^{V 12}$ introduction in HBECs resulted solely in DDR activation (Figure 3a). To examine whether the absence of ARF was due to ARF-mediated p53 apoptosis, flow cytometric detection of cells with sub-G1 DNA-content was employed. An apoptotic wave following Ha-Ras ${ }^{\text {V12 }}$ transfection was noticed, which was prevented by suppressing either ATM or p53 (Figure 3b). Notably, although p53-silencing inhibited apoptosis to a similar extent as ATM knockdown, surviving cells did not show ARF expression (Figures $3 a$ and b). Consistent with the ATM role rather than ARF in apoptosis induction, expression of p53 regulated pro-apoptotic genes Bax, PUMA and NOXA was restored to basal levels after small interfering RNA (siRNA)-mediated ATM silencing, whereas siRNA knockdown of ARF had no effect (Figure $3 \mathrm{c}$ ). Collectively, these results suggest that the lack of ARF expression after one oncogenic 'hit' is not due to p53-mediated apoptosis (that could eliminate the potentially ARF-positive cells), but rather a consequence of low oncogenic load, insufficient to induce ARF.

To examine ARF and DDR in the same human epithelial cell model, yet exposed to higher oncogenic load, we employed the established combination of cell immortalization with telomerase (human telomerase reverse transcriptase (hTERT)) and ectopic mutant Cdk4 expression, followed by the oncogenic 'progression' event achieved by expressing K-Ras ${ }^{112}$.33,34 Analysis revealed that the hTERT/Cdk4 impact was sufficient for DDR activation, whereas ARF expression required the additional oncogenic event from K-Ras ${ }^{V 12}$ (Figure 4a).

Similar results were obtained in BJ fibroblasts, transformed with either $\beta$-catenin, or Ha-Ras ${ }^{V 12}$, or with both. Although 
a

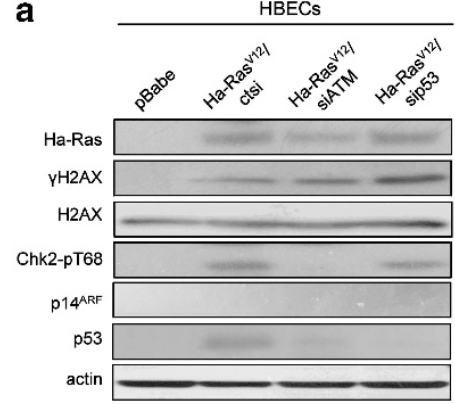

C

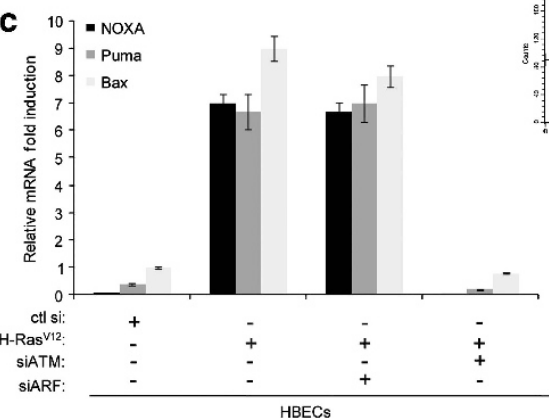

b

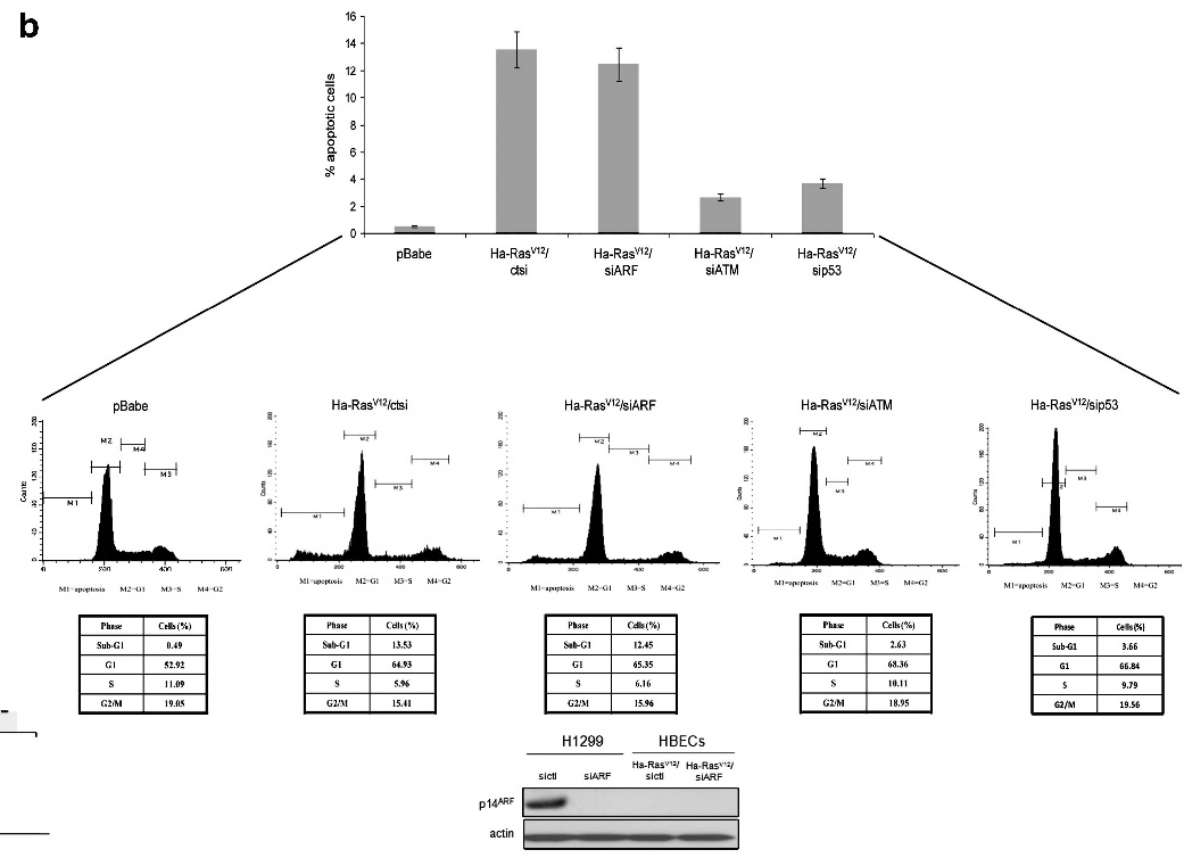

Figure 3 The oncogenic load required for p14 ${ }^{\mathrm{ARF}}$ upregulation is higher compared with DDR activation. (a) Challenging of human bronchial epithelial cells (HBECs) with $H a-R^{V 12}{ }^{V 12}$ leads solely to DDR activation, whereas $\mathrm{p} 14^{\mathrm{ARF}}$ levels are not detected by immunoblot analysis, even when p53 is silenced. (b) Histograms depict an apoptotic wave following Ha-Ras ${ }^{V 12}$ transfection in this setting that is completely abolished by suppressing separately ATM, ARF or p53, as assessed by flow cytometric analysis. Outputs (graphs) from flow cytometric analysis of HBECs, HBECs transfected with Ha-Ras ${ }^{\text {V12 }}$ and HBECs transfected with Ha-Ras ${ }^{V 12}$ followed by ATM, ARF or p53 silencing, demonstrating cell cycle fractions. Successful siRNA of ARF was confirmed in H1299 lung cancer cells that express ARF. (c) ATM silencing restores basal levels for Bax, PUMA and NOXA mRNA (bar graphs from corresponding real-time RT-PCR outputs) in HBECs transiently transfected with Ha-Ras ${ }^{\text {V12 }}$, whereas siARF had no effect

DDR activation was evident in all cases, ARF was induced only upon combined Ha-Ras ${ }^{V 12}$ and $\beta$-catenin expression (Figure 4b). In both HBEC and BJ models of multistep transformation, ARF protein appearance was accompanied by a dramatic raise of its $\mathrm{mRNA}$; supporting a transcription-based ARF induction mechanism in these settings (Figure 4). Increase of ARF at both protein and mRNA levels corresponded with sequential introduction of at least two oncogenic 'hits' suggesting that the late ARF increase, observed during cancer development, is a result of accumulating oncogenic insults.

To validate this, we examined trans-activation of human and murine ARF promoter-driven reporter systems, respectively, in U2OS E2F1-ER cells, combined with ectopic expression of various oncogenes. As documented by reporter data obtained upon expression of various oncogenes, used separately or combined, the reporter signal was significantly amplified when more than one oncogene was activated (Figure 5). Both the human and murine $A R F$ gene promoters contain E2F responsive elements, canonical and non-canonical (Figure $6 a),{ }^{35-38}$ regarded as one of the key downstream effectors of most signaling pathways that trigger ARF transcription. $^{39}$ Of note, Ha-Ras ${ }^{V 12}$ induced transactivation of murine ARF promoter was significantly higher than the human one (Figure 5). This result probably reflects the presence of the DMP1 responsive element in the murine ARF promoter, ${ }^{40}$ pointing-out a species-specific difference in ARF regulation.

To examine whether E2F1 binding to ARF promoter may reflect the increasing oncogenic load in this model system, we performed a chromatin immunoprecipitation (ChIP) assay in the HBEC setting. Concurring with the reporter assays, increased E2F1 binding on ARF promoter was evident only in HBECs that harbored both the active Cdk4 and K-Ras ${ }^{V 12}$ (Figure 6). Notably, the overall endogenous E2F1 protein level was increased about eightfold in cells exposed to higher oncogenic load (Figure 6b), consistent with the induction of ARF in those settings, and the dramatically increased occupation of ARF promoter (Figure 6c).

As these results collectively indicated a major difference between the two barriers in response to a single oncogene, we transformed the BJ fibroblasts with an inducible $\mathrm{Ha}-\mathrm{Ras}^{\mathrm{V12}}$ to study whether prolonged induction of a single oncogene is sufficient for ARF versus DDR induction. Consistent with the emerging requirement for a more robust oncogenic stimulus, ARF remained non-induced, at both protein and mRNA

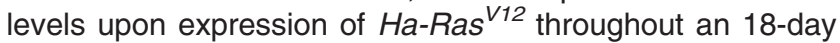
experiment, conditions sufficient to trigger phenotypic features of oncogene-induced senescence (Figures 7a-c). Importantly, the same Ha-Ras expressing BJ-cells showed hallmarks of DDR activation (formation of $\gamma \mathrm{H} 2 \mathrm{AX}$ - or 53BP1-foci), along with p16 ${ }^{\mathrm{INK} 4 \mathrm{~A}}$ and $\mathrm{p} 21^{\mathrm{WAF} 1}$ induction that preceded establishment of senescence (Figures $7 a$ and $b$ ). As positive control, parallel expression in the same BJ cell model of ectopic E2F1, a known transcriptional activator of ARF, 35,41 was sufficient to induce endogenous ARF, along with strong DDR induction (Figures $7 \mathrm{a}$ and $\mathrm{b}$, and data not shown). Despite apoptosis was low in this model (around 1\% as detected by an in situ cytochrom-C release assay), we suppressed p53 to exclude a potential masking of ARF-expressing cells by p53-mediated cell death. Neither ARF protein nor its mRNA, respectively, was elevated upon 
a

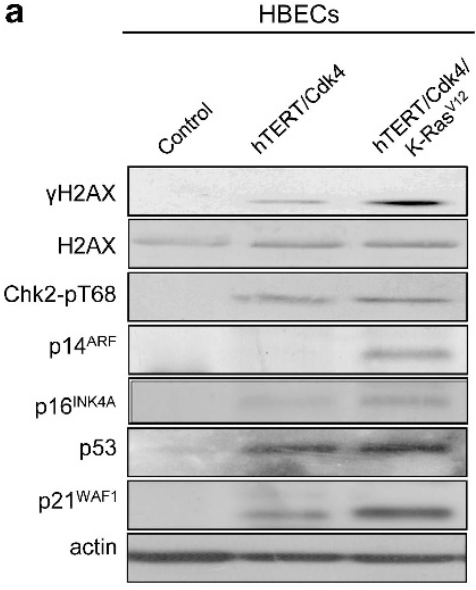

b

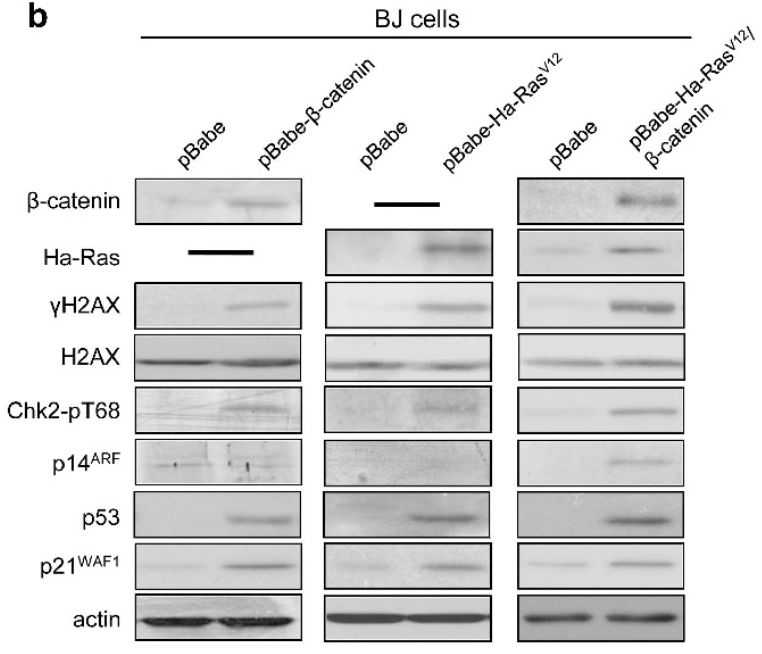

c

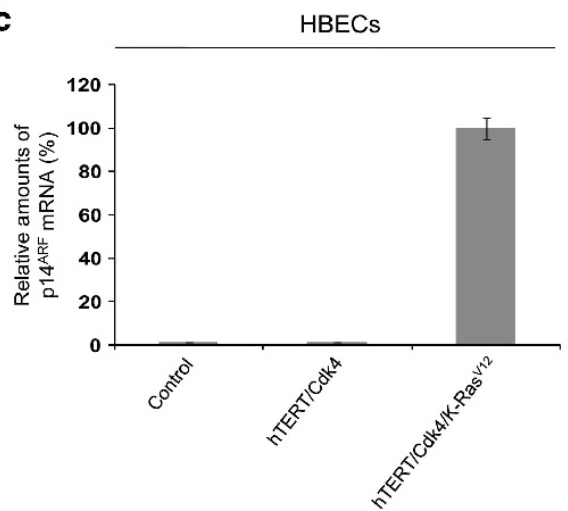

d

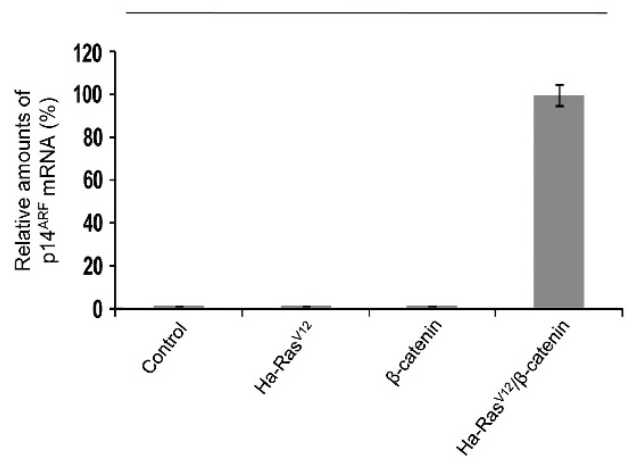

Figure 4 (a) DDR activation precedes $\mathrm{p} 14^{\mathrm{ARF}}$ upregulation upon escalating oncogenic load in HBECs. Immunoblot analysis for DDR markers, p14 ARF along with p53 and

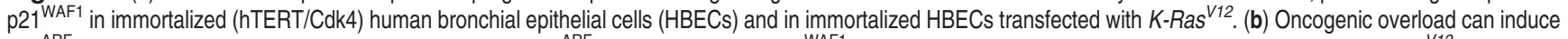
p14 ${ }^{A R F}$ in BJ cells. Immunoblot analysis for DDR markers, p14 ${ }^{A R F}$ along with p53 and p21 ${ }^{\text {WAF1 }}$ in BJ cells transformed with either $\beta$-catenin or activated Ha-Ras ${ }^{V 12}$ or with both $\mathrm{Ha}-\mathrm{Ras}^{\mathrm{V} 12}$ and $\beta$-catenin in serum-starved BJ cells. Serum starvation conditions were indispensable for ARF activation. Actin served as loading control. Oncogene-mediated ARF induction threshold is transcriptionally dependent. Bar graphs from corresponding real-time RT-PCR outputs of p14 ${ }^{A R F} \mathrm{mRNA}$ analysis in: (c) immortalized (hTERT/Cdk4) HBECs, immortalized HBECs transfected with K-Ras ${ }^{V 12}$; (d) human BJ diploid fibroblasts, transformed with either $\beta$-catenin, or activated Ha-Ras ${ }^{V 12}$, or concomitantly with both Ha-Ras ${ }^{V 12}$ and $\beta$-catenin. ARF mRNA levels were clearly detected only upon the sequential introduction of at least two oncogenic 'hits' in both experimental settings. In the experimental systems of $\mathbf{c}$ and $\mathbf{d}$ those with the highest ARF mRNA levels, were arbitrarily set to 1 . All other values were assessed as fold changes relative to these maximal levels, due to the absence of ARF mRNA in certain settings

p53 silencing at any time point throughout these experiments (Figures $7 \mathrm{~d}$ and e). Importantly, the biological impact of p53 silencing in these cells was documented by complete elimination of cellular senescence under conditions with activated Ha-Ras ${ }^{V 12}$ (Figure 7f), thereby excluding the possibility of insufficient p53 knockdown.

As the results obtained so far pointed DDR as a more sensitive 'detector' of oncogenic stress compared with ARF, we further validated this concept by testing whether DDR also contributes a more robust impact in terms of biological responses to threat represented by oncogene-transformed cells. Therefore, we examined the contributions of DDR versus ARF to cellular senescence induced by a single oncogene. Ectopic expression of E2F1 in BJ cells triggered senescence that was bypassed more efficiently by ATM knockdown than depleting ARF (Figure 8a; Supplementary Figure 8). Furthermore, concomitant ATM and ARF silencing did not further strengthen the senescence inhibitory impact compared with depleting ATM alone, supporting the notion that the DDR checkpoint provides the decisive senescence trigger under such conditions (Figure 8a). Next, upon challenging BJ cells with an increased oncogenic load provided by two cooperating oncogenes (Ha-Ras ${ }^{\mathrm{V} 12}$ and $\beta$-catenin, Figure $8 \mathrm{~b}$ ), the percentage of senescent cells was increased compared with those noticed upon expression of either oncogene alone. Under these experimental settings, senescence was bypassed partially upon either selective ATM inhibition or ARF silencing, whereas concomitant ATM and ARF silencing was clearly more potent than inhibiting either pathway alone (Figure 8b). These data suggest that as oncogenic stimuli accumulate, both pathways act in concert to provide a biologically significant defense against increasing threat of malignant transformation.

\section{Discussion}

We provide novel insights into the orchestration and relative contributions of the DDR and ARF tumor-suppressor 
a

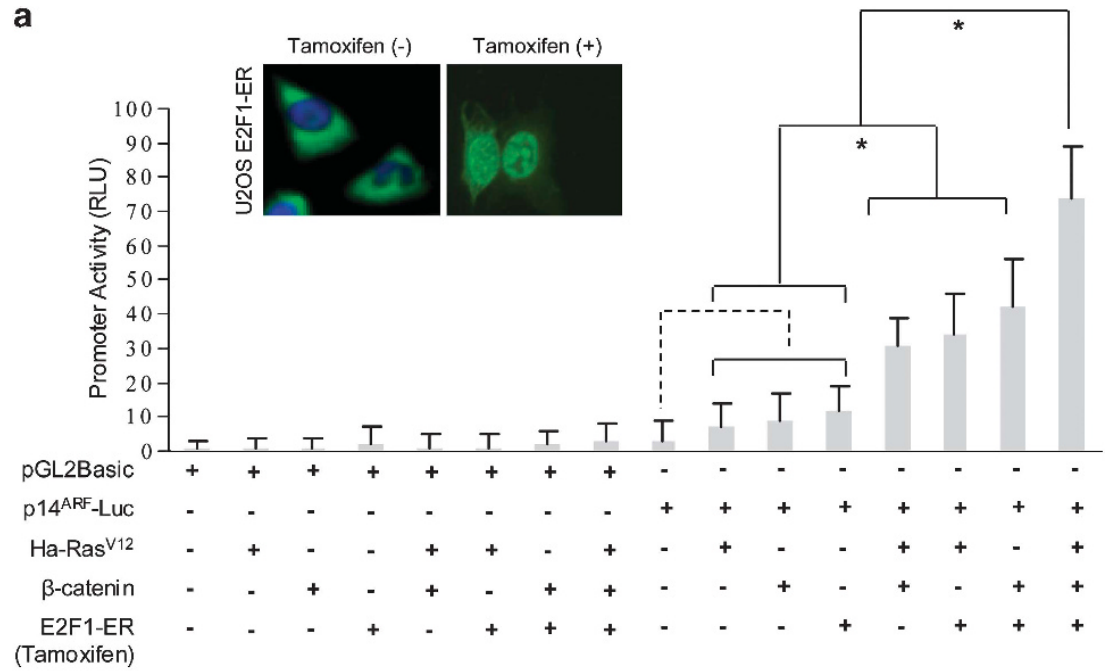

b

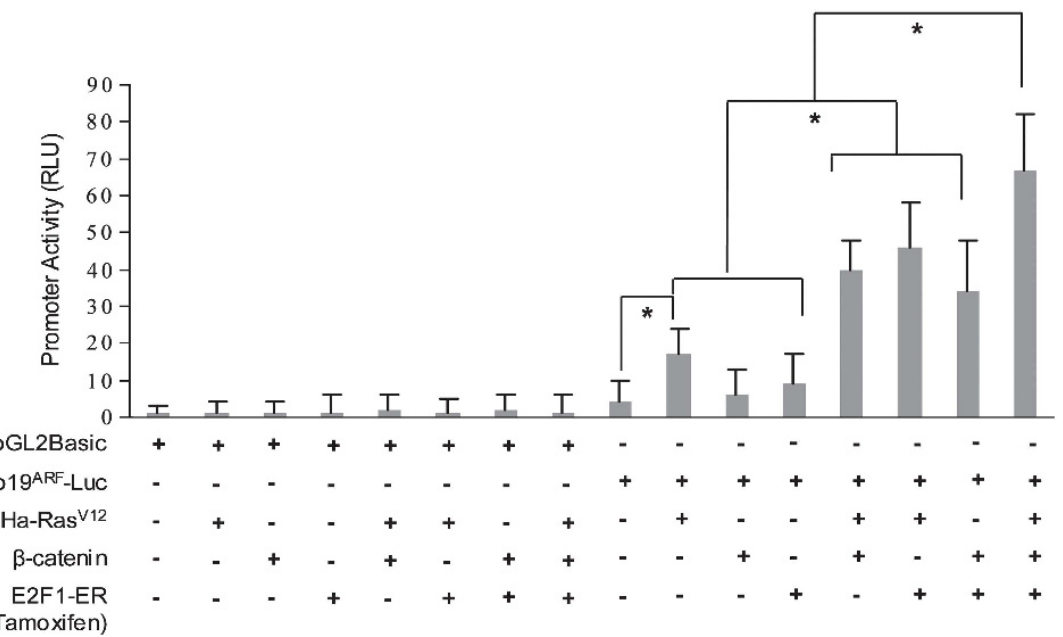

Figure 5 Activation and amplification of ARF reporter signal requires more than one oncogenic 'hits'. Luciferase activity assay of the human (a) (p14ARF-Luc) and murine (b) (pGL2-p19ARF-Luc) ARF promoter-driven luciferase reporters, in U2OS E2F1-ER cells challenged with various oncogenes separately or in conjunction. Note that $\mathrm{Ha}_{\mathrm{R}} \mathrm{RS}^{V 12}$ trans-activates significantly more the murine ARF promoter than the human one. Inset: E2F1 immunofluorescence in U2OS E2F1-ER cells after tamoxifen treatment confirms E2F1 activation (nuclear signal). Statistically significant differences are depicted with * and non-statistically significant as dash lines. RLU, Relative Luciferase Unit

mechanisms. Both respond to oncogenic stress and alarm p53-dependent and -independent antitumor responses. Their respective activation timing, magnitude of response, potential tissue- and species-specific differences, requirements in terms of activation thresholds and relative merits of ARF versus $\mathrm{p} 16^{\mathrm{INK} 4 \mathrm{~A}}$ were addressed. These issues are important not only from mechanistic and conceptual points of view, but also for cancer management. These barriers not only impact cancer progression and, hence, overall incidence of malignancies, but also modulate the outcome of the vast majority of non-surgical treatment modalities used in oncology. This first '4-dimensional' comparative analysis of these mechanisms in multiple experimental and clinical settings, allowed several important and novel conclusions.

First, in vivo analyses of multistage tumorigenesis in mouse models and human epithelial clinical samples showed consistently that ARF is activated later, and less frequently than DDR. This conclusion was based on both parallel immunohistochemical assessments, and wherever feasible by ARF mRNA analysis. ARF protein expression patterns were obtained reproducibly with four different antibodies, consistently with the mRNA analyses. These in vivo data sets were corroborated by functional in vitro analyses, ruling out the possibility that the observed lack of ARF induction in early lesions could reflect insufficiently sensitive staining. Importantly, CDKN2A deletion was excluded as a reason for lack of ARF expression in early lesions, although the locus was inactivated in a subset of the analyzed tumors, consistent with previous reports. ${ }^{29,30}$ These findings indicated that the DDR checkpoint detects oncogenic stress in vivo with higher sensitivity and earlier compared with ARF. Consistently, recent studies of human colon adenomas and carcinomas demonstrated a mutator phenotype ${ }^{42}$ and chromosomal instability, ${ }^{43}$ respectively, with hallmarks of replication stress-induced DNA damage.

Second, considering our initial in vivo data, we employed in vitro human models to test our hypothesis that ARF activation requires more robust oncogenic insult(s), compared 
a
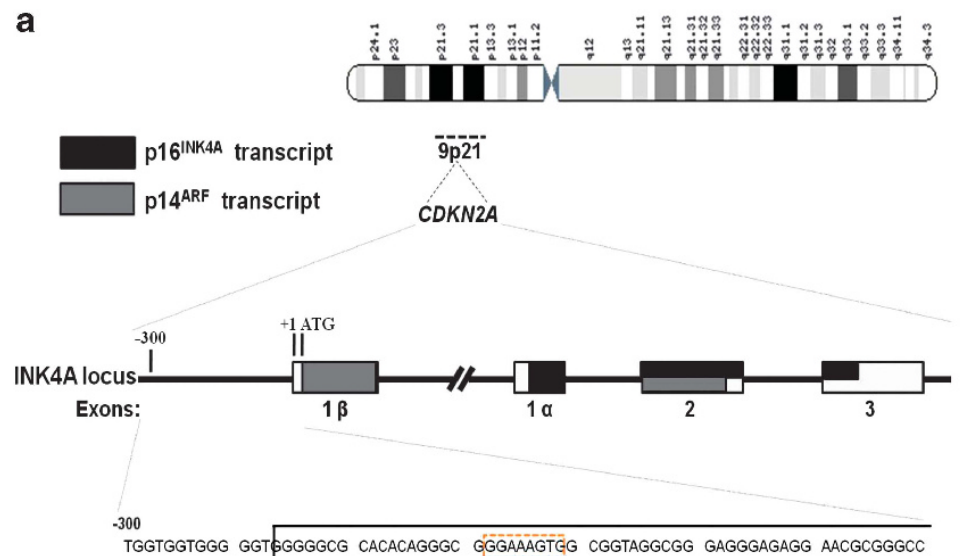

TGGTGGTGGG GGT GGGGGCG CACACAGGGC GGGAAAGTG̈G CGGTAGGCGG GAGGGAGAGG AACGCGGGCC

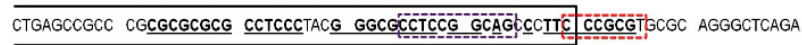

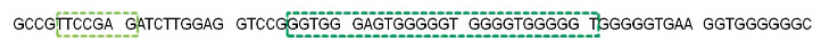

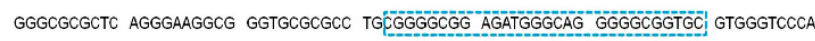

GTCTGCAG AAGGGG̈GCAA GAGTGGCGCT GCTCACCTCT GGTGCCAAAG GGCGGCGCAG CGGCTGCCGA GCTCGGCCCT GGAGGCGGCG AGAACATG

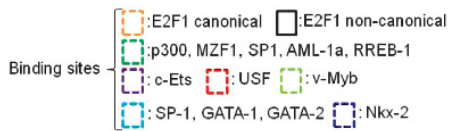

b
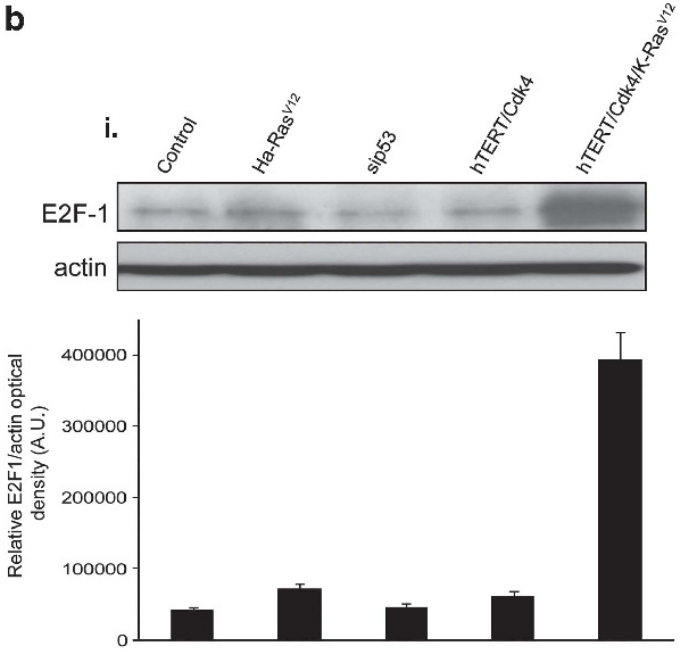

ii.

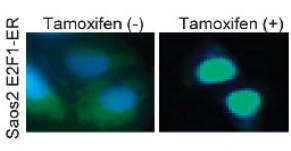

C

PC

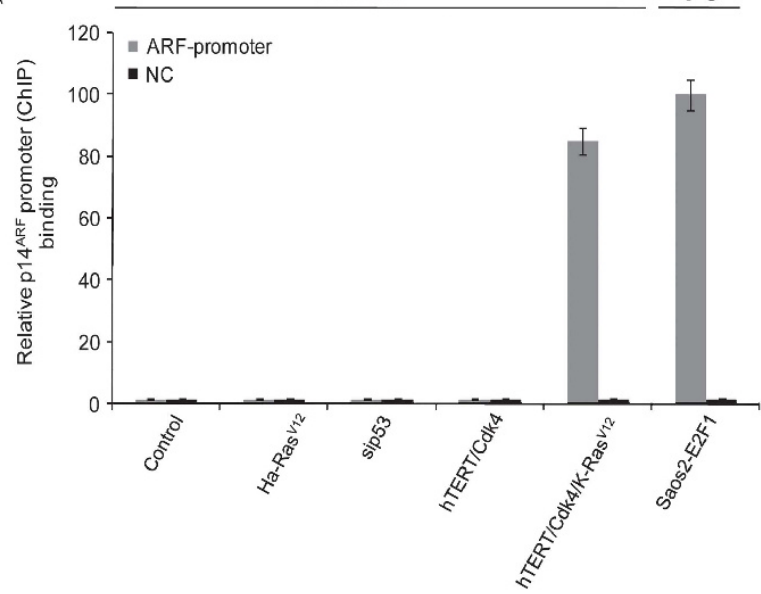

Figure 6 (a) Schema showing the CDKN2A locus and the human ARF promoter on 9p21 chromosome arm. The proximal region ( -300 to 0$)$ of human ARF promoter is depicted at nucleotide level and encompasses a non-canonical E2F1 responsive region located at positions -287 to -180 . Underlined sequence depicts partial homology in the non-canonical E2F-1 region between human and mice. Representative results of a stringent bioinformatic analysis ( $>85 \%$ ) of the region, using the online tool TFSEARCH program, ${ }^{\mathbf{7 5}}$ shows subregions (dashed color lines) that include a number of potential responsive elements to various transcription factors involved in key growth signaling routes. The homology tool scans the TRANSFAC databases (developed at GBF-Braunschweig, Germany) as a source for established transcription factor-binding site profiles. ${ }^{\mathrm{S} 6}$ Increased oncogenic load is followed by elevated E2F1 levels and binding to the human ARF promoter. (bi) Immunoblot analysis shows increased E2F1 levels only in HBECs with both the active Cdk4 and K-Ras ${ }^{\mathrm{V} 12}$. (bii) E2F1 immunofluorescence in Saos2 E2F1-ER cells after tamoxifen treatment confirms E2F1 activation (nuclear signal). (c) Chromatin immunoprecipitation (ChIP) assay (bars from corresponding real-time RT-PCR outputs) reveals binding of E2F1 in the ARF promoter only in the HBECs that harbored both the active Cdk4 and K-Ras ${ }^{\mathrm{V} 12}$. Corresponding values of E2F1 binding on the ARF promoter in Saos2 E2F1-ER cells were used as positive control (PC). They were also arbitrarily set as maximal level (100) relative to which all other fold bindings in the other experimental settings were estimated

with DDR. Indeed, expression of single oncogenes in either BJ-fibroblasts or HBECs was sufficient for induction of DDR and senescence, whereas ARF activation required a combination of oncogenic insults in either cellular model. An intriguing issue related to these results is the nature of molecular triggers capable of activating these pathways. Oncogene-induced replication stress and DNA breakage appear to account for DDR activation, mediated primarily by phosphorylation-dependent signaling, ${ }^{1,3,6,44,45}$ whereas efficient ARF induction reflects a primarily transcription-based mechanism requiring an escalating oncogenic load. The latter finding probably reflects the complex organization of the responsive elements within ARF promoter. ${ }^{35-37}$ As such E2F1, a key ARF inducer, requires the non-canonical responsive region that contains apart to $\mathrm{E} 2 \mathrm{~F} 1$, additional elements for other transcription factors (Figure 6a). Likewise, oncogenic Ras requires endogenous activities of both E2F1 and Sp-1 transcription factors to activate human $A R F^{37}$ Taken together, with our results from various assays, and the elevated ARF and E2F1 levels seen upon combining more than one oncogenic stimulus suggest that a combination of oncogenes signaling through various pathways affecting 
a

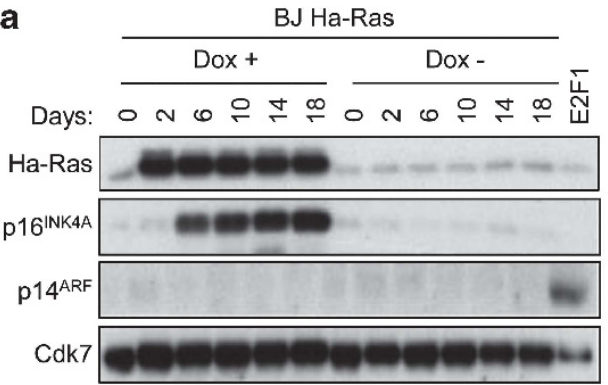

C

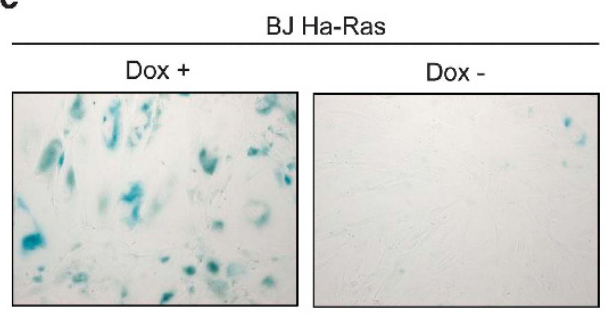

e

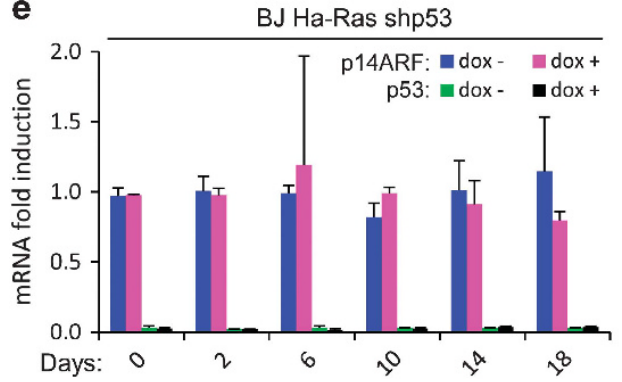

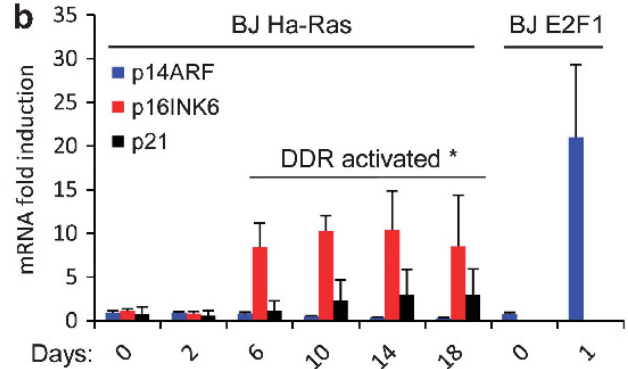

d

BJ Ha-Ras shp53
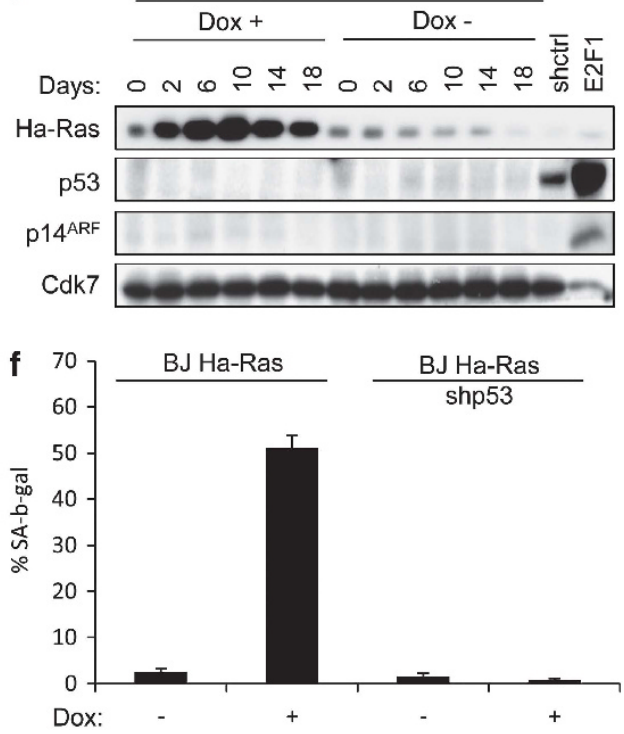

Figure 7 Responses of human BJ cells to prolonged expression of $H a-R a s^{V 12}$ oncogene. (a) Western blots of BJ cell lysates at indicated times during a 18-day timecourse experiment upon induction of $\mathrm{Ha}-\mathrm{Ras}^{\mathrm{V} 12}$ (Dox + ) probed for the indicated proteins. Note the lack of p14 ${ }^{\mathrm{ARF}}$, in contrast to p16 ${ }^{\mathrm{INK} 4 \mathrm{~A}}$. BJ cells with inducible E2F1 were used as positive control for $\mathrm{p} 14^{\mathrm{ARF}}$ expression. (b) p16 $6^{\text {INK4A }}$ and $\mathrm{p} 21^{\mathrm{WAF} 1} \mathrm{mRNA}$ are upregulated after Ha-Ras ${ }^{V 12}$ expression in BJ cells, in contrast to p14 ${ }^{A R F} \mathrm{mRNA}$. Fold change of mRNA is relative to control cells (Dox - , no Ha-Ras ${ }^{\mathrm{V} 12}$ expression). Error bars represent the S.D. of three independent experiments. The Ha-Ras ${ }^{\mathrm{V} 12}$-expressing cells showed enhanced formation of DDR markers: $\gamma \mathrm{H} 2 \mathrm{AX}$ and 53BP1 foci, from day 6 onwards. (c) Senescence-associated SA- $\beta$-gal positivity in Ha-Ras ${ }^{V 12}$-expressing $\left(\right.$ Dox + ) BJ cells compared with control cells, both at day 18. (d) p14 $4^{\mathrm{ARF}}$ is undetectable in BJ cells expressing Ha-Ras ${ }^{\mathrm{V} 12}$ regardless of sh-mediated depletion of $\mathrm{p} 53$ (western blot, 18-day time-course); shctrl vector was used as control for p53 knockdown efficiency, and inducible E2F1 BJ cells were used as positive control for p14 ${ }^{A R F}$ expression. (e) Constitutive depletion of $p 53$ by shRNA ensures low levels of $p 53$ mRNA (green and black graphs), whereas the low basal p14ARF mRNA level (blue, no Ras) remains unchanged despite the expression of $\mathrm{Ha}_{-} \operatorname{Ras}^{V 12}$ (Dox + ; pink) under conditions of $\mathrm{p} 53$ depletion (in both blue and pink bars). Fold change of $p 14^{A R F}$ and p53 mRNA levels are relative to control Dox - (no Ha-Ras ${ }^{V 12}$ ) cells transformed with a control (shctrl) vector (values of 1.0). (f) p53 knockdown leads to bypass of Ha-Ras ${ }^{\text {V12 }}$-induced (Dox + ) senescence (\% SA- $\beta$-gal-positive cells). Dox, doxorubicin

transcription, are required for a biologically meaningful ARF response. This scenario also involves cellular context influence and additional regulation levels, such as feedback loops and post-transcriptional modifications. ${ }^{46-48}$

Third, we addressed the relative contributions of DDR and $A R F$, respectively, to oncogene-evoked cellular senescence or death. ${ }^{1,13,15}$ Consistent with our concept of a dominant DDR role in early responses to limited oncogenic load, the cell-culture experiments showed that both cellular responses (at enhanced level) were attributable to the DDR (ATM)-p53 axis, rather than to ARF. Additionally, in vitro experiments with blocking the p53 function in single-oncogene scenarios, excluded the possibility that potential subsets of ARF expressing cells could be eliminated through ARF/p53-mediated cell death. Even in the only scenario when a single event resulted in ARF induction, namely the ectopic expression of E2F1, ARF expression was largely dispensable for ensuing senescence, in contrast to the causal role of DDR. The intriguing implication of this is that even if ARF is expressed under conditions of a limited oncogenic load, the overall cellular context seems not permissive for ARF to trigger cell-cycle arrest or cell death, and such cellular responses rely on the DDR machinery. Nevertheless, when oncogenic insults accumulate, DDR and ARF act in concert to trigger senescence. Despite sparse evidence showing that an increased oncogenic load is required for induction of senescence or apoptosis, our study is the first that mechanistically demonstrates the co-operation between DDR and ARF under such condition. ${ }^{49,50}$ In case p53, the downstream effector is inactivated, as it usually happens during carcinogenesis, ARF could contribute as an anti-tumor response by exerting the p53 independent functions it possesses. ${ }^{13}$

Fourth, although the bulk in vivo tumorigenesis results obtained were consistent with ARF expression at advanced 


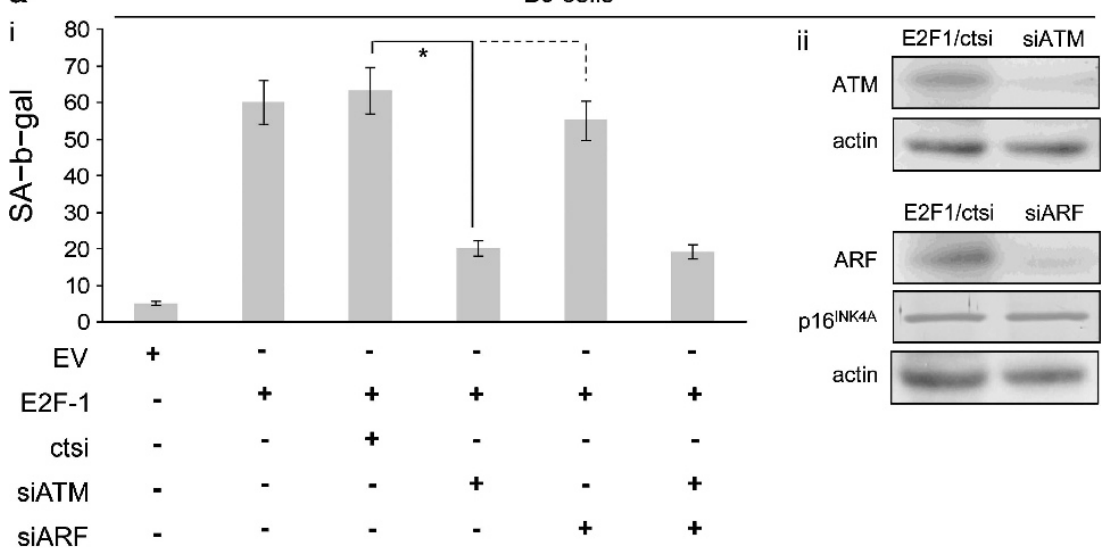

b

BJ cells

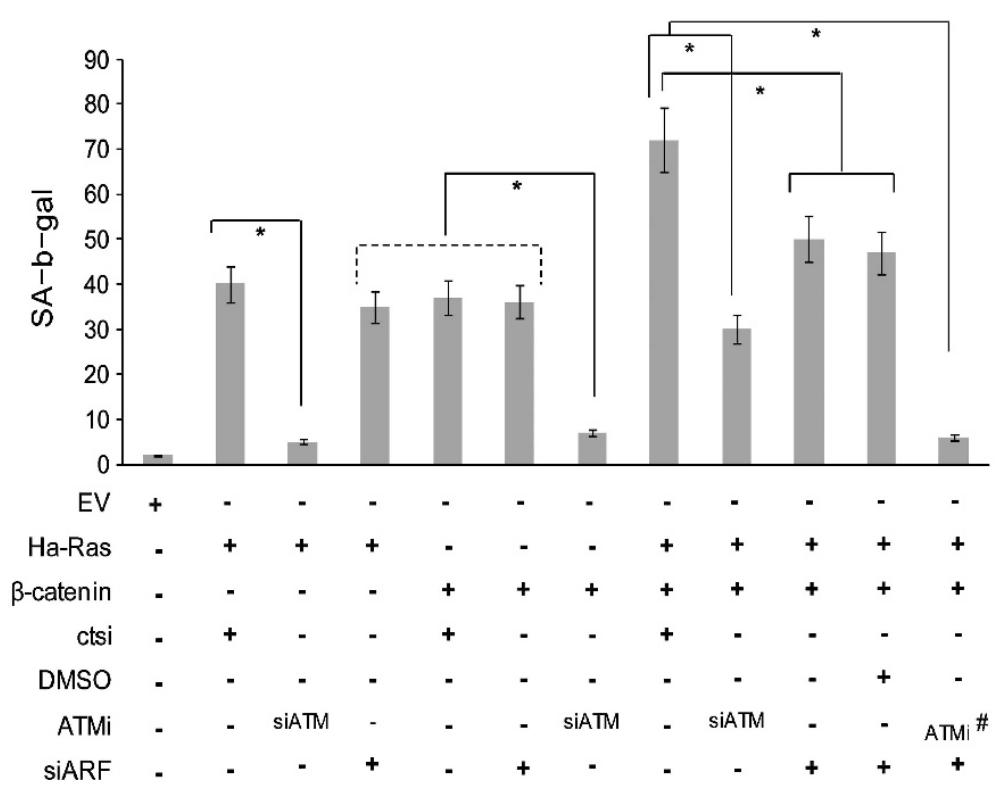

Figure 8 (a) The DDR machinery is more sensitive and more effective than ARF to a single oncogene-mediated antitumor response. (ai) Graph bars denote the percentage of senescent cells (\% SA- $\beta$-gal positive) after corresponding manipulations. Senescence that is induced after E2F1 introduction in BJ cells is bypassed much more efficiently by ATM silencing (SiATM) than ARF inhibition. (aii) Immunoblots showing the efficiency of the anti-ATM and -ARF siRNAs employed and the specificity of the siARF relatively to the $16^{\mathrm{INK} 4 \mathrm{~A}}$. (b) Accumulation of oncogenic stimuli leads the DDR and ARF pathways to act in concert as antitumor responses. Histograms denote the percentage of senescent cells (\% SA- $\beta$-gal positive) after corresponding manipulations. BJ cells challenged with two oncogenes (Ha-Ras ${ }^{12}$ and $\beta$-catenin) exhibit higher proportions of senescent cells, compared with those observed with one oncogene. In this context, senescence is analogously bypassed with either ATM inhibition or ARF silencing. Statistically significant differences are depicted with * and non-statistically significant as dash lines. \# Denotes that ATM inhibition performed either by siATM transfection or treatment with the specific ATM inhibitor Ku55933 revealed similar effects on senescence elimination. Corresponding control experiments showed also no differences in proportions of senescent BJ cells (data not shown)

stages, compared with earlier onset of DDR, we noticed one exception. This was the early detection of human ARF in actinic-keratosis, a precursor for squamous cell skin carcinomas. ${ }^{31}$ Such early ARF expression apparently contradicted the lack of ARF noted in earliest lesions in our nicastrin deficiency model of mouse skin carcinogenesis. This 'exceptional-early' ARF expression in some human skin lesions could be related to the fact that squamous cell skin carcinoma is sunlight related, and the CDKN2 locus is responsive to UV-induced stress. ${ }^{32}$ This interpretation is supported by: (i) the human skin xenograft model of hyperplasias unrelated to UV light; and (ii) the lack of early ARF expression in the mouse model of nicastrin/Notch tumorigenesis. Neither of these settings are UV exposed, and both featured pronounced DDR activation, with undetectable ARF, supporting the concept of delayed ARF activation during tumor progression, and the likely link of heterogeneous ARF expression in the early human skin lesions with UV radiation. As we focused on epithelial tumors that represent the bulk of cancer, it is possible that additional 'exceptions' from the preferential DDR activation compared with ARF may emerge from future tumorigenesis studies in tissues of other histogenesis.

Fifth, another important result, was that $\mathrm{p} 16^{\mathrm{INK} 4 \mathrm{~A}}$, also encoded by the CDKN2 locus, was induced more often than $\mathrm{ARF}$ and earlier during tumor progression in vivo and in in vitro 
models. These results support the partly independent regulation of these overlapping genes ${ }^{2}$ during tumorigenesis, indicating that mainly $\mathrm{p} 16^{\mathrm{INK} 4 \mathrm{~A}}$ expression is likely selected against by $C D K N 2 A$ losses, at least in early human cancer stages. Most of our knowledge regarding INK4/ARF expression concerns $\mathrm{p} 16^{\mathrm{INK} 4 \mathrm{~A}}$ regulation at transcriptional ${ }^{17}$ and post-translational level. ${ }^{51,52}$ Its induction is often associated with mitogen-activated protein kinase activation. ${ }^{53-55}$ The involvement of $\mathrm{p} 16^{\mathrm{INK} 4 \mathrm{~A}}$ from the earliest stages of cancer possibly reflects 'oncogenic' signals triggering the Ras-RafMek pathway. ${ }^{56}$ Ras, a nodal upstream element in this cascade, can lead to $\mathrm{p} 16^{\mathrm{INK} 4 \mathrm{~A}}$ expression by ERK-mediated activation of Ets $1 / 2 .{ }^{57}$ Considering the available literature and our results, the required 'threshold of oncogenic-stress' could again, as in the case of DDR versus ARF, represent the decisive factor underlying the differential $\mathrm{p} 16^{\mathrm{INK} 4 \mathrm{~A}}$ and ARF expression regulation during cancer progression. Finally, as CDKN2A promoter sequences are not well conserved between human and mouse, inter-species differences can be significant. ${ }^{58}$ Indeed, although in rodents relevant stimuli mostly result in co-regulation of the two genes, ${ }^{59,60}$ in humans co-regulation of $\mathrm{p} 16^{\mathrm{INK} 4 \mathrm{~A}}$ and $\mathrm{p} 14^{\mathrm{ARF}}$ is uncommon. ${ }^{17}$

We conclude that in contrast to the earlier, more sensitive and widespread DDR-activation, ARF expression and its biological impact on nascent cancer cells require conditions of escalating oncogenic load, suggesting that ARF may provide a delayed and 'less-sensitive' checkpoint-barrier during cancer development. As events leading to inactivation of these barriers during tumor-progression fuel tumorigenesis at the expense of genome integrity and enhanced dependency on stress-support pathways, this area of cancer research can provide further leads to future therapeutic exploitation of such tumor-selective vulnerabilities. Considering also the complexity and interdependencies of such mechanisms and their changing landscape over time, further elucidation of these pathways, and probably combined targeting of multiple pathways, will be required for more effective and personalized cancer management.

\section{Materials and Methods}

Human samples. Formalin-fixed, paraffin-embedded specimens of normal $(n=7)$ and tumor bladder tissues $(n=103)$ from the University Hospital in Aarhus were examined. The human specimens from the head and neck $(n=19)$, skin $(n=5)$ and pancreas $(n=3)$, comprising the entire spectrum of pathological lesions that develop during epithelial carcinogenesis, were obtained from the Laboratory of Histology and Embryology, Athens Medical School. All samples were collected according to local ethical committee approvals. None of the patients had undergone any cancer therapy before surgical resection of the lesions.

Mouse models. The mouse model of human skin xenografts employed in this study was previously described. ${ }^{9}$

UPII/Ha-Ras-M transgenic mice expressing a constitutively active rabbit $\mathrm{Ha}-\mathrm{Ras}^{Q 61 L}$ under the control of a $3.6-\mathrm{kb}$ mouse UPII promoter were produced as reported before. ${ }^{18, \mathrm{~S} 61}$

Mice with colitis-associated colon cancer were obtained by intraperitoneal injection of AOM (10 mg/kg in phosphate-buffered saline), followed after 1 week by three cycles of DSS treatment. Each cycle comprised oral administration of $2 \%$ DSS; $\left(w / v\right.$ in $\left.\mathrm{H}_{2} \mathrm{O}\right)$ for 7 days and subsequent administration of $\mathrm{H}_{2} \mathrm{O}$ for 14 days. Mice were killed at 12 weeks after $\mathrm{AOM}$ injection and colon tissue preparations were performed by standard histological manipulations.

PdxCre;LSL-K-Ras ${ }^{D 12}$ mice, constantly expressing the oncogenic, constitutive active, form of K-Ras in all pancreatic progenitors, under the PdxCre activation, were employed. ${ }^{\mathbf{S 6 2 , \mathbf { S 6 3 }}}$ The animals were anesthetized with isofluarane and avertin, perfused with ice-cold $4 \%$ paraformaldehyde and the tissues were further fixed with $4 \%$ paraformaldehyde for $5 \mathrm{~h}$ at $4{ }^{\circ} \mathrm{C}$. Paraffin blocks were obtained.

Nicastrin conditional knockout $\left(\mathrm{Ncsth}^{\mathrm{f} / /}\right)$ mice $^{\mathrm{S} 64}$ were crossed to mice ubiquitously expressing a CRE-ERT2 transgene (ROSA26 CRE-ERT2/CRE-ERT2) ${ }^{\mathrm{S65}}$ and the Ncstn ${ }^{\mathrm{f} /+}$; ROSA26 $6^{\mathrm{CRE}-\mathrm{ERT} 2 /+}$ offspring was backcrossed to the Ncstn ${ }^{\mathrm{f} / \mathrm{f}}$ parents, to produce $\mathrm{Ncstn}^{\mathrm{f} / \mathrm{f}}$; ROSA26 $6^{\mathrm{CRE}-\mathrm{ERT} 2 /+}$ offspring. Six- to eight-week-old mice were administered $3 \mathrm{mg}$ of tamoxifen citrate, orally through a feeding needle, every other day for a total of four doses, in order to produce mice in which the Ncstn locus is disrupted in all tissues ( $\mathrm{Ncstn}^{-1}-$ ). Four to six weeks after tamoxifen citrate administration, mice were killed and tissues were either snap frozen in liquid nitrogen or fixed in $10 \%$ formalin $\mathrm{O} / \mathrm{N}$ at $4{ }^{\circ} \mathrm{C}$ and embedded in paraffin for sectioning.

Immunohistochemistry. Antibodies and immunohistochemistry analysis were essentially performed as previously described. ${ }^{7-9}$ Details are provided in Supplementary Information.

Cell culture and treatments. Normal human BJ fibroblasts, BJ cells with inducible E2F1 and BJ TetON-Ha-Ras ${ }^{\text {V12 }}$ cells were maintained in Dulbecco's modified Eagle's medium (DMEM; Biochrome, Bioline, Athens, Greece), Biochrome is the manufacturer and Bioline is the local distributor, with $10 \%$ FCS (Biochrome, Bioline, Athens, Greece), $2 \mathrm{mM}$ L-glutamine (Biochrome, Bioline,), $100 \mu \mathrm{g} / \mathrm{ml}$ penicillin and streptomycin (Biochrome, Bioline), respectively, at $37^{\circ} \mathrm{C}$ and $5 \% \mathrm{CO}_{2}$. U2OS E2F1-ER and Saos2 E2F1-ER cells were cultured as previously described. ${ }^{\text {S66 }}$

Normal HBECs, immortalized (hTERT/Cdk4) HBECs and immortalized HBECs transfected with K-Ras ${ }^{V 12} \cdot{ }^{34}$ All HBEC variants were grown in keratinocyte serum-free medium (Invitrogen, Carlsbad, CA, USA) supplemented with $50 \mu \mathrm{g} / \mathrm{ml}$ bovine pituitary extract and $5 \mathrm{ng} / \mathrm{ml} \mathrm{hEGF} \mathrm{(Invitrogen),} \mathrm{at} 37^{\circ} \mathrm{C}$ and $5 \% \mathrm{CO}_{2}{ }^{34}$

Cells at $80 \%$ confluency were treated with $10 \mu$ M ATM inhibitor (Ku55933, Merck, Athens, Greece) ${ }^{\mathrm{s} 67}$ or DMSO for $24 \mathrm{~h}$.

Inducible cell lines, plasmids, retrovirus construction and infections. Stable human BJ fibroblast strain conditionally expressing Ha-Ras ${ }^{V 12}$ was generated by cloning Ha-Ras ${ }^{\mathrm{V} 12}$ under the control of a Tet-dependent promoter (Tet-On Advanced; Clontech, Mountain View, CA, USA). The system contains two elements, the regulator vector (pLVX-Tet-On Advanced) and the response vector (pLVX-Tight-Puro). Two recombinant lentiviruses (pLVX-Tet-On Advanced and pLVX-Tight-Puro) were produced using three other helper vectors pLP-1, pLP-2 and pVSVG (Invitrogen) following previously described protocol. ${ }^{\mathrm{S} 68} \mathrm{BJ}$ cells infected with both recombinant lentivirus were selected with $2 \mu \mathrm{g} / \mathrm{ml}$ puromycin and $500 \mu \mathrm{g} / \mathrm{ml} \mathrm{G} 418$. Expression of $\mathrm{Ha}-\mathrm{Ras}^{V 12}$ was regulated by adding of into the medium at a final concentration $2 \mu \mathrm{g} / \mathrm{ml}$.

Vector for p53 knockdown in BJ fibroblast strain conditionally expressing $\mathrm{Ha}$-Ras ${ }^{\mathrm{V} 12}$ was generated by cloning hygromycin cassette from vector pLKO.1 hygro (Addgene plasmid 24150) via BamHI and Kpnl to replace the puromycin cassette in shp53 pLKO.1 puro (Addgene plasmid 19119). Lentivirus for p53 knockdown was generated using vectors pMD2.G (Addgene plasmid 12259) and psPAX2 (Addgene plasmid 12260) following previously described protocols. ${ }^{\mathbf{6} 9}$

Retrovirus for preparation of E2F1-expressing BJ cells was generated by transfection of Phoenix cells with pBabeHAERE2F (kindly provided by Kristian Helin, University of Copenhagen) using the calcium phosphate method. BJ cells infected with retrovirus for $72 \mathrm{~h}$ were selected with $2 \mathrm{mg} / \mathrm{ml}$ puromycin. Expression of $\mathrm{N}$-terminally HA-tagged ER-E2F1 was regulated by the addition of 4-hydroxytamoxifen at a final concentration of $300 \mathrm{nM}$. The functionality of this E2F1 construct was reported previously. ${ }^{7}$

Transient infections with pBabe- $\beta$-catenin, ${ }^{\mathrm{S} 70}$ pBabe-Ha-Ras ${ }^{\mathrm{V} 12}$, pBabe-E2F1 and the corresponding control vector using the Phoenix helper-free retrovirus producer cell line were performed as previously described. ${ }^{\mathrm{S} 66}$ In brief, $70 \%$ confluent Phoenix cells were transfected, using the Lipofectamine 2000 transfection kit (Invitrogen), with the gene expressing vectors or the control vector alone, and the obtained replication-incompetent retroviruses were used for the infection of HBECs, BJ or U2OS E2F1-ER cells along with $10 \mu \mathrm{g} / \mathrm{ml}$ polybrene (Sigma, Athens, Greece). Twenty-four hours after infection, the cells were selected with the appropriate antibiotic for 2 days and harvested for further analysis 2 days later, unless otherwise specified (Supplementary Figure 8). For serum starvation conditions, $24 \mathrm{~h}$ after infection, culture medium was replaced with DMEM containing $0.1 \%$ serum with the addition of the appropriate antibiotics. Selection lasted for $48 \mathrm{~h}$ and the cells were cultured for another $48 \mathrm{~h}$ with the low-serum-containing DMEM. 
Transfections with control siRNA (Stealth RNAi, Invitrogen), anti-ATM (Stealth RNAi, Invitrogen), anti-p14 ${ }^{A R F}$ (Invitrogen) or anti-p53 (kind gift from Dr. Moshe Oren) siRNA pools, as well as shp53, applying Effectene (Qiagen), were performed as previously described. ${ }^{11, \mathrm{~S} 66}$

Luciferase reporter assay. Luciferase reporter assays were performed as

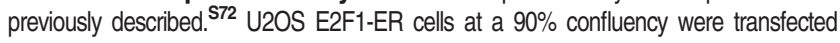
using $50 \mu \mathrm{l}$ OptiMEM-reduced serum medium, $1 \mu \mathrm{l} \mathrm{Lipofectamine} 2000$ (Invitrogen) and $0.2 \mu \mathrm{g}$ of plasmid pGL2Basic/Luc, p14ARF-Luc (kind gift of Dr. PP Pandolfi) or pGL2p19ARF-Luc (kind gift of Dr. PP Pandolfi), along with $0.2 \mu \mathrm{g}$ of pBabe-Ha-Ras ${ }^{V 12}$ or pBabe- $\beta$-catenin or combination of both, respectively, as described in previous section. Four hours before cell collection, E2F1 nuclear translocation was tamoxifen induced (Supplementary Figure 8). ${ }^{7}$ Cell extracts were obtained as recommended by the manufacturer (Luciferase Assay System E1500, Promega, Madison, WI, USA). Luc activities were measured in a Safire2 TECAN microplate reader (Tecan, Männedorf Switzerland). Transfection efficiency was estimated by co-transfecting the $\mathrm{pCH} 110$ plasmid containing a lacZ gene expressed under the control of the SV40 early promoter (GE HealthCare, Athens, Greece). All transfections were repeated three times.

Indirect immunofluorescence. Indirect immunofluorescence analysis was performed according to a published protocol ${ }^{11}$ and is described in details in the Supplementary Information.

ChIP assay. ChIP assays were performed as previously described. ${ }^{\mathrm{S71}}$ Detailed description is provided in Supplementary Information.

Senescence associated $\boldsymbol{\beta}$-galactosidase (Sa- $\beta$-gal) staining. BJ cells were infected with control, Ha-Ras ${ }^{\mathrm{V} 12}$, shp53, $\beta$-catenin or E2F1 pBabe vectors as well as combinations of them (Figure 8 and Supplementary Figures 7 and 8 ) as described in previous section, except selection that lasted for 4 days. Subsequently, cells grown on coverslips were transfected with anti-ATM or anti-ARF siRNAs and/or treated with an ATM chemical inhibitor (Ku55933 as described in previous section) or DMSO. Finally, at day 10, control and treated cells were fixed in $1 \%$ paraformaldehyde, processed for Sa- $\beta$-gal activity and counterstained with nuclear fast red, as described elsewhere. ${ }^{9} \mathrm{BJ}$ cells conditionally expressing $\mathrm{Ha}-\mathrm{Ras}^{\mathrm{V} 12}$ were fixed at day 18 and stained with Senescence $\beta$-Galactosidase Staining Kit (Cell Signalling, Bioline, \#9860). Only cytoplasmic staining was scored as a positive signal.

Protein extraction and immunoblotting. Protein extraction and immunoblotting analysis were essentially performed as previously described ${ }^{11}$ and is presented in detail in Supplementary Information.

Flow cytometric analysis. Flow cytometric analysis was conducted as previously described. ${ }^{11}$ Details are provided in Supplementary Information.

Isolation of nucleic acids. DNA extraction and RNA isolation were performed as described elsewhere. ${ }^{11}$ See details in Supplementary Information.

cDNA preparation and real-time reverse transcription (RT)-PCR analysis. CDNA was generated using the Superscript II Reverse Transcriptase (Invitrogen) and oligo-dT (Invitrogen) following the supplier's guidelines.

Evaluation of $p 14^{A R F}, p 16^{\text {INKAA }}$, Bax, Puma and Noxa mRNA status was performed by real-time RT-PCR analysis ${ }^{11}$ using the Platinum SYBR Green qPCR SuperMix-UDG (Invitrogen) according to the manufacturer's instructions. PCR reactions were performed on a DNA-Engine-Opticon (MJ-Research, Athens, Greece) thermal cycler. Two reference genes, GAPDH and $P B G D$, were used to validate the results. Primer sequences and annealing temperatures are provided in Supplementary Table 3 . The results are presented as fold changes against untreated or positive control samples, accordingly. The mean value was calculated from three independent measurements.

Allelic imbalance analysis of microsatellite loci D9S161. Allelic imbalance analysis was performed as previously described ${ }^{\mathrm{ST2}}$ and is presented in detail in Supplementary Information.

K-Ras mutational analysis. K-Ras mutation status at codons 12 and 13 was established by using PCR-RFLP combined with Sanger sequencing as previously published. ${ }^{\mathrm{S} 3}$ Details are presented in Supplementary Information.
Bladder cancer DNA copy number and gene expression data. DNA copy number analysis of CDKN2A locus was performed in 39 bladder tumors using Affymetrix SNP 6.0 arrays (Affymetrix, Santa Clara, CA, USA). CDKN2A transcripts were profiled in 11 biopsies of normal urothelium and in 44 bladder tumors using Affymetrix Exon 1.0 ST arrays by standard protocols. Normalized gene expression measures were generated using GCRMA in GeneSpring 10.0 (Agilent Technologies Inc, Santa Clara, CA, USA). The probe sets 3201480 and 3201481 are specific for measuring $p 14^{A R F}$ and the probe sets 3201464-67 are specific for measuring $\mathrm{p} 16^{\mathrm{INK} 4 \mathrm{~A}}$ levels. Probe detection above background was used in order to exclude non-functional probe sets before analysis. An average value for the two $\mathrm{p} 14^{\mathrm{ARF}}$ probe sets was used.

We used the CRMAv2 method from the R package aroma.affymetrix for the preprocessing and probe summarization of the SNP6.0 data for CDKN2A copy number estimation. This method produces full-resolution raw copy number estimates by the following steps: calibration for crosstalk between allele probe pairs, normalization for 25-mer nucleotide-position probe sequence effects, robust probe summarization (probe for the two alleles are summed) and calculation of full resolution total copy number by looking at the ratio of the probe summaries between the tumor and its matched germline or the average of all normal samples. Only the autosomal chromosomes were processed. Before segmentation, we removed the probes belonging to known copy number polymorphisms. Next, we used the $\mathrm{R}$ package, Rseg ${ }^{\mathrm{S7}}$ to segment each tumor sample. Noise was reduced by using a 5-marker median smoothing, and histograms of pre-segmented arrays were used to define thresholds for calling gains and losses and to correct for the shift of the no change peak induced by normalization in case of unbalance between gains and losses.

Statistical analysis. ANOVA and Mann-Whitney tests were employed for statistical analysis. All calculations were performed with the SPSS 17.0 software.

\section{Conflict of Interest}

The authors declare no conflict of interest.

Acknowledgements. We thank Proffessor M Oren for kindly providing the CM5 antibody and the anti-p53 siRNA, Dr. PP Pandolfi for kindly donating plasmids pGL2-p19ARF-Luc and p14ARF-Luc, Dr. Robert Strauss for donating plasmids shp53 pLKO.1 hygro and Dr. A Damalas and Antonia Daleziou for their technical support. Financial support was from the Danish Cancer Society, the Danish National Research Foundation, the European Commission FP7 projects: GENICA, INFLA-CARE, BioMedReg, DDResponse, INsPiRE and the NKUA-SARG projects (codes: 11351 and 8916). VG and MP are recipients of a Hellenic Association for Molecular Cancer Research scholarship, whereas Dr. A Kotsinas received an Empeirikeion Foundation fellowship.

1. Halazonetis TD, Gorgoulis VG, Bartek J. An oncogene-induced DNA damage model for cancer development. Science 2008; 319: 1352-1355.

2. Gil J, Peters G. Regulation of the INK4b-ARF-INK4a tumour suppressor locus: all for one or one for all. Nat Rev Mol Cell Biol 2006; 7: 667-677.

3. Gorgoulis VG, Halazonetis TD. Oncogene-induced senescence: the bright and dark side of the response. Curr Opin Cell Biol 2010; 22: 816-827.

4. Collado $M$, Serrano $M$. Senescence in tumours: evidence from mice and humans. Nat Rev Cancer 2010; 10: 51-57.

5. Zilfou JT, Lowe SW. Tumor suppressive functions of p53. Cold Spring Harb Perspect Biol 2009; 1: a001883.

6. Negrini S, Gorgoulis VG, Halazonetis TD. Genomic instability-an evolving hallmark of cancer. Nat Rev Mol Cell Biol 2010; 11: 220-228.

7. Bartkova J, Horejsí Z, Koed K, Krämer A, Tort F, Zieger K et al. DNA damage response as a candidate anti-cancer barrier in early human tumorigenesis. Nature 2005; 434: 864-870.

8. Gorgoulis VG, Vassiliou LV, Karakaidos P, Zacharatos P, Kotsinas A, Liloglou T et al. Activation of the DNA damage checkpoint and genomic instability in human precancerous lesions. Nature 2005; 434: 907-913.

9. Bartkova J, Rezaei N, Liontos M, Karakaidos P, Kletsas D, Issaeva $\mathrm{N}$ et al. Oncogene-induced senescence is part of the tumorigenesis barrier imposed by DNA damage checkpoints. Nature 2006; 444: 633-637.

10. Di Micco R, Fumagalli M, Cicalese A, Piccinin S, Gasparini P, Luise $C$ et al. Oncogene-induced senescence is a DNA damage response triggered by DNA hyper-replication. Nature 2006; 444: 638-642. 
11. Liontos M, Koutsami M, Sideridou M, Evangelou K, Kletsas D, Levy B et al. Deregulated overexpression of hCdt1 and hCdc6 promotes malignant behavior. Cancer Res 2007; 67 10899-10909.

12. Sherr CJ, Weber JD. The ARF/p53 pathway. Curr Opin Genet Dev 2000; 10: 94-99.

13. Sherr CJ. Ink4-Arf locus in cancer and aging. Wiley Interdiscip Rev Dev Biol 2012; 1 731-741.

14. Hoeijmakers JH. Genome maintenance mechanisms for preventing cancer. Nature 2001; 411: $366-374$.

15. Jackson SP, Bartek J. The DNA-damage response in human biology and disease. Nature 2009; 461: 1071-1078.

16. Branzei D, Foiani M. Maintaining genome stability at the replication fork. Nat Rev Mol Cell Biol 2010; 11: 208-219.

17. Kim WY, Sharpless NE. The regulation of INK4/ARF in cancer and aging. Cell 2006; 127 265-275.

18. Mo L, Zheng X, Huang HY, Shapiro E, Lepor H, Cordon-Cardo $\mathrm{C}$ et al. Hyperactivation of Ha-ras oncogene, but not Ink4a/Arf deficiency, triggers bladder tumorigenesis. J Clin Invest 2007; 117: 314-325

19. Wirtz S, Neufert C, Weigmann B, Neurath MF. Chemically induced mouse models of intestinal inflammation. Nat Protoc 2007; 2: 541-546.

20. Chen J, Huang XF. The signal pathways in azoxymethane-induced colon cancer and preventive implications. Cancer Biol Ther 2009; 8: 1313-1317.

21. Wang QS, Papanikolaou A, Nambiar PR, Rosenberg DW. Differential expression of p16(INK4a) in azoxymethane-induced mouse colon tumorigenesis. Mol Carcinog 2000; 28: 139-147.

22. Ekbom A, Helmick C, Zack M, Adami HO. Ulcerative colitis and colorectal cancer. N Engl J Med 1990; 323: 1228-1233.

23. Borinstein SC, Conerly M, Dzieciatkowski S, Biswas S, Washington MK, Trobridge P et al. Aberrant DNA methylation occurs in colon neoplasms arising in the azoxymethane colon cancer model. Mol Carcinog 2010; 49: 94-103.

24. Peng DF, Kanai Y, Sawada M, Ushijima S, Hiraoka N, Kitazawa S et al. DNA methylation of multiple tumor-related genes in association with overexpression of DNA methyltransferase 1 (DNMT1) during multistage carcinogenesis of the pancreas. Carcinogenesis 2006; 27 1160-1168.

25. Artavanis-Tsakonas S, Rand MD, Lake RJ. Notch signaling: cell fate control and signal integration in development. Science 1999; 284: 770-776

26. Nicolas M, Wolfer A, Raj K, Kummer JA, Mill P, van Noort M et al. Notch1 functions as a tumor suppressor in mouse skin. Nat Genet 2003; 33: 416-421.

27. Sarkar S, Jülicher KP, Burger MS, Della Valle V, Larsen CJ, Yeager TR et al. Different combinations of genetic/epigenetic alterations inactivate the p53 and pRb pathways in invasive human bladder cancers. Cancer Res 2000; 60: 3862-3871.

28. Vikhanskaya F, Kei Lee M, Mazzoletti M, Broggini M, Sabapathy K. Cancer-derived p53 mutants suppress p53-target gene expression - potential mechanism for gain of function of mutant p53. Nucleic Acids Res 2007; 35: 2093-2104.

29. Schulz WA. Understanding urothelial carcinoma through cancer pathways. Int J Cancer 2006; 119: 1513-1518.

30. Makitie AA, Monni O. Molecular profiling of laryngeal cancer. Expert Rev Anticancer The 2009; 9: 1251-1260.

31. Yanofsky VR, Mercer SE, Phelps RG. Histopathological variants of cutaneous squamous cell carcinoma. J Skin Cancer 2011; 2011: 210813

32. Rünger TM, Vergilis I, Sarkar P, DePinho RA, Sharpless NE. How disruption of cell cycle regulating genes might predispose to sun-induced skin cancer. Cell Cycle 2005; 4 : 643-645.

33. Ramirez RD, Sheridan S, Girard L, Sato M, Kim Y, Pollack J et al. Immortalization of human bronchial epithelial cells in the absence of viral oncoproteins. Cancer Res 2004; 64 : 9027-9034.

34. Sato M, Vaughan MB, Girard L, Peyton M, Lee W, Shames DS et al. Multiple oncogenic changes (K-RAS(V12), p53 knockdown, mutant EGFRs, p16 bypass, telomerase) are no sufficient to confer a full malignant phenotype on human bronchial epithelial cells. Cancer Res 2006; 66: 2116-2128.

35. Robertson KD, Jones PA. The human ARF cell cycle regulatory gene promoter is a $\mathrm{CpG}$ island which can be silenced by DNA methylation and down-regulated by wild-type p53. Mol Cell Biol 1998; 18: 6457-6473.
36. Inoue K, Roussel MF, Sherr CJ. Induction of ARF tumor suppressor gene expression and cell cycle arrest by transcription factor DMP1. Proc Natl Acad Sci USA 1999; 96: 3993-3998.

37. Berkovich $E$, Lamed $Y$, Ginsberg D. E2F and Ras synergize in transcriptionally activating p14ARF expression. Cell Cycle 2003; 2: 127-133.

38. Tsantoulis PK, Gorgoulis VG. Involvement of E2F transcription factor family in cancer. Eur J Cancer 2005; 41: 2403-2414.

39. del Arroyo AG, El Messaoudi S, Clark PA, James M, Stott F, Bracken A et al. E2F-dependent induction of p14ARF during cell cycle re-entry in human T cells. Cell Cycle 2007; 6: 2697-2705.

40. Inoue K, Mallakin A, Frazier DP. Dmp1 and tumor suppression. Oncogene 2007; 26: 4329-4335.

41. Bates S, Phillips AC, Clark PA, Stott F, Peters G, Ludwig RL et al. p14ARF links the tumour suppressors RB and p53. Nature 1998; 395: 124-125.

42. Nikolaev SI, Sotiriou SK, Pateras IS, Santoni F, Sougioultzis S, Edgren H et al, A single-nucleotide substitution mutator phenotype revealed by exome sequencing of human colon adenomas. Cancer Res 2012; 72: 6279-6689.

43. Burrell RA, McClelland SE, Endesfelder D, Groth P, Weller MC, Shaikh N et al. Replication stress links structural and numerical cancer chromosomal instability. Nature 2013; 494: 492-496.

44. Bartek J, Mistrik M, Bartkova J. Thresholds of replication stress signaling in cancer development and treatment. Nat Struct Mol Biol 2012; 19: 5-7.

45. Bermejo R, Lai MS, Foiani M. Preventing replication stress to maintain genome stability: resolving conflicts between replication and transcription. Mol Cell 2012; 45 : 710-718.

46. Stott FJ, Bates S, James MC, McConnell BB, Starborg M, Brookes S et al. The alternative product from the human CDKN2A locus, p14(ARF), participates in a regulatory feedback loop with p53 and MDM2. EMBO J 1998; 17: 5001-5014.

47. Berkovich E, Ginsberg D. Ras induces elevation of E2F-1 mRNA levels. J Biol Chem 2001; 276: 42851-42856.

48. Haupt Y. Certainly no ARF terthought: oncogenic cooperation in ARF induction a key step in tumor suppression. Cell Cycle 2003; 2: 113-115.

49. Sarkisian CJ, Keister BA, Stairs DB, Boxer RB, Moody SE, Chodosh LA. Dose-dependent oncogen-induced senescence in vivo and its evasion during mammary tumorigenesis. Nat Cell Biol 2007; 9: 493-505.

50. Murphy DJ, Junttila MR, Pouyet L, Karnezis A, Shchors K, Bui DA et al. Distinct thresholds govern Myc's biological output in vivo. Cancer Cell 2008; 14: 447-457.

51. Zhu D, Xu G, Ghandhi S, Hubbard K. Modulation of the expression of p16INK4a and p14ARF by hnRNP A1 and A2 RNA binding proteins: Implications for cellular senescence. J Cell Physiol 2002; 193: 19-25.

52. Lal A, Kim HH, Abdelmohsen K, Kuwano Y, Pullmann R Jr, Srikantan S et al. p16INK4a translation suppressed by miR-24. PLoS One 2008; 3: e1864.

53. Iwasa $\mathrm{H}$, Han J, Ishikawa F. Mitogen-activated protein kinase p38 defines the common senescence-signalling pathway. Genes Cells 2003; 8: 131-144.

54. Bulavin DV, Phillips C, Nannenga B, Timofeev O, Donehower LA, Anderson CW et al. Inactivation of the Wip1 phosphatase inhibits mammary tumorigenesis through p38 MAPK-mediated activation of the p16Ink4a-p19 Arf pathway. Nat Genet 2004; 36: 343-350.

55. Ito K, Hirao A, Arai F, Takubo K, Matsuoka S, Miyamoto K et al. Reactive oxygen species act through p38 MAPK to limit the lifespan of hematopoietic stem cells. Nat Med 2006; 12 : 446-451.

56. Chang L, Karin M. Mammalian MAP kinase signaling cascades. Nature 2001; 410: 37-40.

57. Ohtani N, Zebedee Z, Huot TJ, Stinson JA, Sugimoto M, Ohashi Y et al. Opposing effects of Ets and Id proteins on p16INK4a expression during cellular senescence. Nature 2001; 409: 1067-1070.

58. Ortega S, Malumbres M, Barbacid M. Cyclin D-dependent kinases, INK4 inhibitors and cancer. BBA-Rev Cancer 2002; 1602: 73-87.

59. Zindy F, Quelle DE, Roussel MF, Sherr CJ. Expression of the p16(INK4a) tumor suppressor versus other INK4 family members during mouse development and aging. Oncogene 1997; 15: 203-211.

60. Krishnamurthy J, Torrice C, Ramsey MR, Kovalev Gl, Al-Regaiey K, Su L et al. Ink4a/Arf expression is a biomarker of aging. $J$ Clin Invest 2004; 114: 1299-1307.

Supplementary Information accompanies this paper on Cell Death and Differentiation website (http://www.nature.com/cdd) 